\title{
Estimates of BMO type for singular integrals on spaces of homogeneous type and applications to hypoelliptic PDEs
}

\section{Marco Bramanti and Luca Brandolini}

\begin{abstract}
Let us consider the class of "nonvariational uniformly hypoelliptic operators":

$$
L u \equiv \sum_{i, j=1}^{q} a_{i j}(x) X_{i} X_{j} u
$$

where: $X_{1}, X_{2}, \ldots, X_{q}$ is a system of Hörmander vector fields in $\mathbb{R}^{n}$ $(n>q),\left\{a_{i j}\right\}$ is a $q \times q$ uniformly elliptic matrix, and the functions $a_{i j}(x)$ are continuous, with a suitable control on the modulus of continuity. We prove that:

$$
\left\|X_{i} X_{j} u\right\|_{B M O\left(\Omega^{\prime}\right)} \leq c\left\{\|L u\|_{B M O(\Omega)}+\|u\|_{B M O(\Omega)}\right\}
$$

for domains $\Omega^{\prime} \subset \subset \Omega$ that are regular in a suitable sense. Moreover, the space $B M O$ in the above estimate can be replaced with a scale of spaces of the kind studied by Spanne. To get this estimate, several results are proved, regarding singular and fractional integrals on general spaces of homogeneous type, in relation with function spaces of $B M O$ type.
\end{abstract}

\section{Introduction}

Let $L$ be a linear second order nonvariational uniformly elliptic operator in $\mathbb{R}^{n}$,

$$
L u \equiv \sum_{i, j=1}^{n} a_{i j}(x) u_{x_{i} x_{j}} .
$$

2000 Mathematics Subject Classification: Primary 35H10; Secondary 43A85, 42B20.

Keywords: BMO, hypoelliptic operators, singular integrals, spaces of homogeneous type. 
It is well known that, if $u$ solves the problem

$$
\begin{cases}L u=f & \text { in } \Omega \\ u=0 & \text { on } \partial \Omega\end{cases}
$$

where $\Omega$ is a bounded smooth domain of $\mathbb{R}^{n}$ and $a_{i j}$ are continuous on $\bar{\Omega}$, then

$$
\left\|u_{x_{i} x_{j}}\right\|_{L^{p}(\Omega)} \leq c\|f\|_{L^{p}(\Omega)} \text { for any } p \in(1, \infty)
$$

(see, e.g. [20], p.242). Moreover, Chiarenza-Frasca-Longo ([13], [14]) have shown that the continuity condition can be relaxed assuming $a_{i j} \in L^{\infty}(\Omega) \cap$ $V M O(\Omega)$. These $L^{p}$ estimates are known to fail for $p=\infty$ and $p=1$, even for the Laplace operator. A fairly natural question is to ask whether the John-Nirenberg space $B M O$ and the Hardy space $H^{1}$ can, respectively, replace $L^{\infty}$ and $L^{1}$ in similar estimates. (Throughout this introduction, the reader is assumed to be familiar with the definition of $B M O, V M O$ and related spaces. However, all the background will be given in detail in $\S 1$ ).

In 1966, Peetre [27] proved the following local result for elliptic operators: if $L$ is as above, and its coefficients have a modulus of continuity which is $o(1 /|\log t|)$, then for every test function $u$ supported in a ball small enough,

$$
\left\|u_{x_{i} x_{j}}\right\|_{*} \leq c\left\{\|L u\|_{*}+\left\|u_{x_{i} x_{j}}\right\|_{2}\right\}
$$

where $\|\cdot\|_{*}$ is the $B M O$ seminorm. This estimate exploits another Peetre's result of independent interest, namely the continuity of a singular integral operator of Calderón-Zygmund type on $B M O$.

In 1993, Chang-Krantz-Stein [11] proved global estimates in suitable Hardy spaces $H^{p}$ for the second derivatives of solutions to boundary value problems on bounded domains for the Laplacian.

In 1999, Chang-Dafni-Stein [9] improved the above $H^{p}$ estimates and established $B M O$ estimates, in the same context (BVPs for the Laplacian).

In the same year, Chang-Li [10] studied $H^{1}$ and $B M O$ estimates for the second derivatives of solutions to the Dirichlet problem for elliptic operators with Dini-continuous coefficients. These results partially overlap with those of Peetre, who proves only local results, but on the other side has a slightly weaker assumption on the coefficients.

The aim of this paper is to extend to spaces of homogeneous type (of finite measure) Peetre's theorem on $B M O$ continuity of Calderón-Zygmund operators, and several related results, and apply these to prove local $B M O$ regularity estimates for the class of "uniformly hypoelliptic operators" studied by the authors in [4], [5]. 
More precisely we consider operators of the form

$$
L u \equiv \sum_{i, j=1}^{q} a_{i j}(x) X_{i} X_{j} u
$$

where: $X_{1}, X_{2}, \ldots, X_{q}$ is a system of Hörmander vector fields in $\mathbb{R}^{n}(n>q)$, the $q \times q$ matrix $\left\{a_{i j}\right\}$ is symmetric and uniformly elliptic, and the functions $a_{i j}(x)$ satisfy a continuity assumption of the kind assumed by Peetre. We shall prove that:

$$
\left\|X_{i} X_{j} u\right\|_{B M O\left(\Omega^{\prime}\right)} \leq c\left\{\|L u\|_{B M O(\Omega)}+\|u\|_{B M O(\Omega)}\right\}
$$

for any bounded domain $\Omega$, and $\Omega^{\prime} \subset \subset \Omega\left(\Omega, \Omega^{\prime}\right.$ satisfying a suitable regularity assumption). More generally, we shall prove estimates of type (0.2), with $B M O$ replaced by a scale of spaces (introduced by Spanne [32]), which in particular contains $B M O$ (see $\S 1$ for the definition of these spaces, and $\S 4.2$ for the exact statement of this estimate). Here the $B M O$-type spaces are defined with respect to the Carnot-Carathéodory distance induced by vector fields.

To motivate our assumptions on the coefficients $a_{i j}(x)$ and the techniques we employ to prove $(0.2)$, let us come back again to discuss the elliptic case. Since the $B M O$ estimate can be seen as an endpoint case of $L^{p}$ estimates, in view of Chiarenza-Frasca-Longo's results, it would seem natural to work with discontinuous coefficients $a_{i j}$, belonging (at least) to $L^{\infty} \cap V M O$.

To follow this approach one needs the following two estimates:

$$
\begin{gathered}
\|K f\|_{B M O} \leq c\|f\|_{B M O} \\
\left\|\left[K, M_{a}\right] f\right\|_{B M O} \leq c\|a\|\|f\|_{B M O}
\end{gathered}
$$

where $K$ is a singular integral operator, $\left[K, M_{a}\right] f=K(a f)-a K(f)$, and $\|a\|$ is some seminorm of $a$ which is locally small when $a$ has small oscillations (in some suitable sense). Now, the best estimate of kind (0.3) that we actually are able to prove (see $\S 5$ ) involves the $L^{2} M O$ seminorm for $a$ :

$$
\|a\|=\sup _{B_{r}} \frac{\log ^{2} r}{\left|B_{r}\right|} \int_{B_{r}}\left|a(x)-a_{B_{r}}\right| d x .
$$

But the assumption of finiteness of this $\|a\|$ implies continuity of $a$, as we shall see in $\S 1.2$. Since, on the other side, the $L^{2} M O$ assumption seems very natural from the point of view of the techniques involved in the proof of (0.3), we are led to think that it is better to assume a priori the continuity of the $a_{i j}$ 's. At this point the Chiarenza-Frasca-Longo commutator technique 
is no longer natural to attack the problem: instead, it is natural (and much easier!) to apply the classical "Korn's trick" (see [23]). Let us sketch this idea in the case of a variable coefficient elliptic operator

$$
L u \equiv \sum_{i, j=1}^{n} a_{i j}(x) u_{x_{i} x_{j}}
$$

For a test function $u$ supported in some ball $B_{r}\left(x_{0}\right)$, we consider the frozen operator

$$
L_{0} u \equiv \sum_{i, j=1}^{n} a_{i j}\left(x_{0}\right) u_{x_{i} x_{j}}
$$

Then for a suitable singular integral operator $K$ we have the representation formula

$$
u_{x_{i} x_{j}}(x)=K\left(L_{0} u\right)(x)=K(L u)(x)+\sum_{i, j=1}^{n} K\left(\left[a_{i j}\left(x_{0}\right)-a_{i j}(\cdot)\right] u_{x_{i} x_{j}}\right)(x) .
$$

Now we exploit the $B M O$ continuity of $K$ proved by Peetre and the fact, proved by Stegenga [33] and $\mathrm{Li}$ [24], that

$$
\|a f\|_{*} \leq c\left\{\|a\|_{L M O}+\|a\|_{\infty}\right\}\|f\|_{*}
$$

where

$$
\|a\|_{L M O}=\sup _{B_{r}} \frac{|\log r|}{\left|B_{r}\right|} \int_{B_{r}}\left|a(x)-a_{B_{r}}\right| d x .
$$

Then

$$
\begin{aligned}
\left\|u_{x_{h} x_{k}}\right\|_{*} & \leq c\left\{\|L u\|_{*}+\sum_{i, j}\left\|\left[a_{i j}\left(x_{0}\right)-a_{i j}(\cdot)\right] u_{x_{i} x_{j}}\right\|_{*}\right\} \leq \\
& \leq c\left\{\|L u\|_{*}+\sum_{i, j}\left(\left\|a_{i j}\right\|_{L M O}+\left\|a_{i j}\left(x_{0}\right)-a_{i j}\right\|_{\infty}\right)\left\|u_{x_{i} x_{j}}\right\|_{*}\right\} .
\end{aligned}
$$

Now, if $u$ is supported in a ball $B_{r}$ small enough, and the coefficients $a_{i j}$ have a suitable modulus of continuity, then the term

$$
\left(\left\|a_{i j}\right\|_{L M O}+\left\|a_{i j}\left(x_{0}\right)-a_{i j}\right\|_{\infty}\right)
$$

vanishes with $r$, and one can derive the $B M O$ estimates. These estimates hold under assumptions that are weaker than asking $a_{i j} \in L^{2} M O$, and only require the study of the multiplication operator on $B M O$ (rather than the commutator).

If one is willing to translate this technique to the setting of hypoelliptic operators of kind (0.1), what is needed is to prove, in a suitable general context, a $B M O$ continuity result for singular (and fractional) integral operators, and a theorem stating that the multiplication for a maps $B M O$ continuously to itself, whenever $a \in L^{\infty} \cap L M O$. 
These facts will be proved here for general spaces of homogeneous type of finite measure and will imply, as we shall explain in $§ 4.2$, an estimate of the kind

$$
\left\|X_{i} X_{j} u\right\|_{B M O\left(B_{r}\right)} \leq c\left\{\|L u\|_{B M O\left(B_{r}\right)}+\|u\|_{B M O\left(B_{r}\right)}\right\}
$$

for any function $u$ compactly supported in $B_{r}$ and $r$ small enough. From this we deduce (0.2), exploiting suitable interpolation inequalities for "Sobolev" spaces, and several properties related to the geometry induced by the vector fields, which will be established in $\S \S 4.1-4.2$.

To summarize, the main results in this paper are divided in two groups:

a) General results which hold in a space of homogeneous type, of finite measure: Theorem 2.4 (multiplication operator on spaces of $B M O$ type), Theorem 3.4 (singular integrals on spaces of $B M O$ type), Theorem 3.5 (fractional integrals on spaces of $B M O$ type), and Theorem 5.1 (commutator of a singular integral with the multiplication operator).

b) Applications to Hörmander's vector fields and "non-variational uniformly hypoelliptic operators": Theorem 4.5 (local BMO type estimates), Theorems 4.13 and 4.15 (interpolation inequality for Sobolev norms defined by vector fields). We also point out some properties of the space $B M O$ on a bounded domaim, proved in paragraph 4.1.

The proof of Theorem 4.5 relies on all the other results we have just quoted, with the exception of Theorem 5.1, which we have inclosed in the Appendix only for the sake of completeness.

\section{Definitions and known results}

\subsection{Spaces of homogeneous type}

Let $X$ be a set. A function $d: X \times X \rightarrow \mathbb{R}$ is called a quasidistance on $X$ if there exists a constant $c_{d} \geq 1$ such that for any $x, y, z \in X$ :

$$
\begin{gathered}
d(x, y) \geq 0 \text { and } d(x, y)=0 \Leftrightarrow x=y \\
d(x, y)=d(y, x) \\
d(x, y) \leq c_{d}(d(x, z)+d(z, y)) .
\end{gathered}
$$

We will say that two quasidistances $d, d^{\prime}$ on $X$ are equivalent, and we will write $d \simeq d^{\prime}$, if there exist two positive constants $c_{1}, c_{2}$ such that $c_{1} d^{\prime}(x, y) \leq$ $d(x, y) \leq c_{2} d^{\prime}(x, y)$ for any $x, y \in X$. 
For $r>0$, let $B_{r}(x)=\{y \in X: d(x, y)<r\}$. These "balls" satisfy the axioms of complete system of neighborhoods in $X$, and therefore induce a (separated) topology in $X$. With respect to this topology, the balls $B_{r}(x)$ need not to be open. We will explicitly exclude the above kind of pathology:

Definition 1.1 Let $(X, d)$ be a set endowed with a quasidistance d such that the d-balls are open with respect to the topology induced by $d$, and let $\mu$ be a positive Borel measure on $X$ satisfying the doubling condition: there exists a positive constant $c_{\mu}$ such that

$$
\mu\left(B_{2 r}(x)\right) \leq c_{\mu} \cdot \mu\left(B_{r}(x)\right) \text { for any } x \in X, r>0 .
$$

Then $(X, d, \mu)$ is called a space of homogeneous type.

To simplify notation, the measure $d \mu(x)$ will be denoted simply by $d x$, and $\mu(A)$ will be written $|A|$. This will not create confusion because throughout the paper we will consider only one measure. We will also set

$$
B(x ; y)=B_{d(x, y)}(x) .
$$

The above definition of space of homogeneous type is the one introduced by Coifman-Weiss [16].

Remark 1.2 In some applications it is natural to consider quasidistances $d$ that satisfy a quasisymmetric condition, weaker than (1.1):

$$
d(x, y) \leq c_{d} d(y, x) .
$$

A way to bypass the problem is to set $d^{\prime}(x, y)=d(x, y)+d(y, x)$ obtaining an equivalent quasidistance $d^{\prime}$ that satisfies the symmetry property $d^{\prime}(x, y)=$ $d^{\prime}(y, x)$.

The main drawback of property (1.2), compared with the standard triangle inequality, is that it prevents us from writing

$$
\left|d(x, y)-d\left(x_{0}, y\right)\right| \leq d\left(x, x_{0}\right) .
$$

This, for instance, is why we cannot prove in general that the balls are open. The lack of the above inequality is partially compensated by a useful property of quasidistances:

Theorem 1.3 (Macias-Segovia, see [25]) If $d$ is a quasidistance in $X$, there exists another quasidistance $d^{\prime}$, equivalent to $d$, such that

$$
\left|d^{\prime}(x, y)-d^{\prime}\left(x_{0}, y\right)\right| \leq c d^{\prime}\left(x, x_{0}\right)^{\alpha}\left[d^{\prime}(x, y)^{1-\alpha}+d^{\prime}\left(x_{0}, y\right)^{1-\alpha}\right]
$$

for some constants $c>0, \alpha \in(0,1]$, any $x_{0}, x, y \in X$. We say that $d^{\prime}$ is of order $\alpha$.

In view of the above property, without loss of generality we can assume that $d$ is already of order $\alpha$ (for some $\alpha \in(0,1]$ ). 
Throughout the paper we will consider spaces of homogeneous type of finite measure. By the doubling property, this requirement is equivalent to the boundedness of the space (see $[6]$ ).

\subsection{Function spaces}

Let $(\Omega, d, d x)$ be a space of homogeneous type of finite measure.

The function spaces we are going to define will depend on a function $\varphi:[0,+\infty) \rightarrow[0,+\infty)$, which from now on will be assumed to satisfy the following conditions:

i: $\varphi$ is nondecreasing;

ii: $\varphi$ is doubling:

$$
\varphi(2 t) \leq c \varphi(t) \quad \forall t>0 ;
$$

iii: $\varphi$ satisfies the "logarithmic vanishing estimate":

$$
\frac{\varphi(t r)}{\varphi(r)} \geq \frac{c}{(1+|\log t|)^{\beta}}
$$

for every $r>0, t \in(0,1)$, some positive constants $c, \beta$.

Note that iii is also equivalent to the following, which will be useful sometimes:

$$
\frac{\varphi(r)}{\varphi\left(2^{-k} r\right)} \geq \frac{c}{(1+k)^{\beta}}
$$

for any positive integer $k, r>0$, some positive constants $c, \beta$.

Set:

$$
\begin{aligned}
\operatorname{BMO}_{\varphi}(\Omega)=\left\{f \in L^{1}(\Omega):\right. & \|f\|_{*, \varphi, \Omega}= \\
& \left.\sup _{x \in \Omega, r>0} \frac{1}{\left|B_{r}(x)\right| \varphi(r)} \int_{B_{r}}\left|f(y)-f_{B_{r}}\right| d y<\infty\right\},
\end{aligned}
$$

where

$$
f_{B_{r}}=\frac{1}{\left|B_{r}\right|} \int_{B_{r}} f(y) d y
$$

(When $\Omega$ is implicitly understood, we will write simply $\|f\|_{*, \varphi}$ ). Since $\Omega$ is bounded, there exists $R$ such that for every $x_{0} \in \Omega$ and $r \geq R$ we have $B_{r}\left(x_{0}\right)=\Omega$. We will call $R$ the diameter of $\Omega$. Note that, in the above definition, taking the supremum for $x \in \Omega, r>0$ is the same as taking the supremum for $x \in \Omega, 0<r \leq R$. 
For $\varphi \equiv 1,\|\cdot\|_{*, \varphi}$ coincides with the standard BMO seminorm, denoted by $\|\cdot\|_{*}$, and we get the classical space "Bounded Mean Oscillation", introduced by John-Nirenberg in [22]. Note that

$$
\|f\|_{*, \varphi} \geq \frac{1}{\varphi(R)}\|f\|_{*}
$$

that is

$$
B M O_{\varphi}(\Omega) \subset B M O(\Omega) \subset L^{p}(\Omega) \quad \text { for every } p<\infty .
$$

(For the general properties of $B M O$ on spaces of homogeneous type, see [8]). Let:

$$
\|f\|_{B M O \varphi}=\|f\|_{*, \varphi}+\|f\|_{L^{1}(\Omega)} .
$$

We also set $\operatorname{LMO}(\Omega)=B M O_{\varphi}(\Omega)$ if $\varphi=1 /|\log r|$ for small $r$. More precisely, if $R$ is as above, then $\operatorname{LMO}(\Omega)$ is defined by

$$
\varphi(r)= \begin{cases}\frac{1}{\log _{2} \frac{2 R}{r}} & \text { for } 0<r \leq R \\ 1 & \text { for } r>R .\end{cases}
$$

Analogously, $L^{2} M O(\Omega)=B M O_{\varphi}(\Omega)$ with $\varphi=1 / \log ^{2} r$ for small $r$.

Spanne [32] proves (in the Euclidean case) that if $\varphi$ satisfies the Dini condition

$$
\int_{0}^{\delta} \frac{\varphi(t)}{t} d t<\infty
$$

then functions in $\mathrm{BMO}_{\varphi}(\Omega)$ are continuous, with a modulus of continuity

$$
\omega(r) \leq c \int_{0}^{r} \frac{\varphi(t)}{t} d t
$$

Conversely, if $\varphi$ is an increasing function such that (1.6) fails, then there exist unbounded discontinuous functions belonging to $B M O_{\varphi}(\Omega)$. These properties still hold in a space of homogeneous type. By Spanne's criterion, functions in $L^{2} M O$ are continuous, while functions in $L M O$ may be discontinuous.

For $f \in B M O_{\varphi}(\Omega)$, set

$$
\eta(f, \varphi, \Omega)(r)=\sup _{x \in \Omega, \rho \leq r} \frac{1}{\left|B_{\rho}(x)\right| \varphi(\rho)} \int_{B_{\rho}}\left|f(y)-f_{B_{\rho}}\right| d y .
$$

Then, $V M O$-type spaces are defined by:

$$
\operatorname{VMO}_{\varphi}(\Omega)=\left\{f \in \mathrm{BMO}_{\varphi}(\Omega): \eta(f, \varphi, \Omega)(r) \rightarrow 0 \text { for } r \rightarrow 0\right\} .
$$

When $B_{M}(\Omega)$ equals $B M O, L M O, L^{2} M O$, the spaces $V M O_{\varphi}$ will be denoted, respectively, by $V M O, V L M O, V L^{2} M O$. We recall that $V M O$ stands for "Vanishing Mean Oscillation", the space introduced by Sarason in [31]. 


\section{Preliminary computations. The multiplication operator}

Let $\varphi$ be as in the previous section, and denote by $R$ the diameter of $\Omega$. The following functions, built up on $\varphi$, well be useful:

$$
\begin{aligned}
S_{\varphi}(r) & =\int_{r}^{2 R} \frac{\varphi(t)}{t} d t, \\
\widetilde{\varphi}(r) & =\frac{\varphi(r)}{1+S_{\varphi}(r)}
\end{aligned}
$$

for $r \in[0, R]$.

Lemma 2.1 The function $S_{\varphi}$ is nonincreasing on $[0, R]$; the function $\widetilde{\varphi}$ satisfies conditions $i$, ii, iii of $\S 1.2$.

The proof is a straightforward computation.

Lemma 2.2 For any $f \in B M O_{\varphi}(\Omega), r \leq R$, we have

$$
\left|f_{B_{r}}\right| \leq\left|f_{\Omega}\right|+c\|f\|_{*, \varphi} \sum_{k=1}^{\left[\log _{2}(2 R / r)\right]} \varphi\left(2^{k} r\right)
$$

with $c$ independent of $f$ (where [.] denotes the integral part).

Proof. By the doubling condition

$$
\begin{aligned}
\left|f_{B_{2^{j}}}-f_{B_{r}}\right| & \leq \sum_{k=1}^{j}\left|f_{B_{2^{k_{r}}}}-f_{B_{2^{k-1} r}}\right| \leq c \sum_{k=1}^{j} f_{B_{2^{k_{r}}}}\left|f-f_{B_{2^{k_{r}}}}\right| \\
& =c \sum_{k=1}^{j} \frac{\varphi\left(2^{k} r\right)}{\varphi\left(2^{k} r\right)} f_{B_{2^{k_{r}}}}\left|f-f_{B_{2^{k}}}\right| \leq c \sum_{k=1}^{j} \varphi\left(2^{k} r\right)\|f\|_{*, \varphi} .
\end{aligned}
$$

Now, for $r<R$, let $j$ be the positive integer such that $2^{j-1} r \leq R<2^{j} r$, so that $j=\left[\log _{2} \frac{2 R}{r}\right]$, then $f_{B_{2 j_{r}}}=f_{B_{R}}$ and

$$
\left|f_{B_{r}}\right| \leq\left|f_{B_{R}}\right|+\left|f_{B_{2 j_{r}}}-f_{B_{r}}\right| \leq\left|f_{B_{R}}\right|+c\|f\|_{*, \varphi} \sum_{k=1}^{\left[\log _{2} \frac{2 R}{r}\right]} \varphi\left(2^{k} r\right) .
$$

From the first line of the proof of the previous Lemma we also read the following useful relation:

$$
\left|f_{B_{2^{j}}}-f_{B_{r}}\right| \leq c j\|f\|_{*}
$$

with $c$ independent of $j, r$. 
Another computation shows that:

Lemma 2.3 There exist constants $c^{\prime}, c^{\prime \prime}$ such that for every $r \leq R$,

$$
c^{\prime} S_{\varphi}(r) \leq \sum_{k=1}^{\left[\log _{2} \frac{2 R}{r}\right]} \varphi\left(2^{k} r\right) \leq c^{\prime \prime} S_{\varphi}(r) .
$$

In view of Lemmas 2.2-2.3, we can write the following estimate, which will be useful several times:

$$
\frac{\left|f_{B_{r}}\right|}{\varphi(r)} \leq c \frac{\|f\|_{B M O_{\varphi}}}{\widetilde{\varphi}(r)}
$$

Let $M_{a}: f \mapsto a f$. We now want to study under which assumptions on $a$ the multiplication operator $M_{a}$ is continuous on $B M O_{\varphi}(\Omega)$. We will get a generalization of the results proved by Stegenga [33] and Li [24].

Theorem 2.4 If $a \in L^{\infty} \cap B M O_{\widetilde{\varphi}}(\Omega)$, then $M_{a}$ maps $B M O_{\varphi}(\Omega)$ into itself continuously. More precisely:

$$
\|a f\|_{*, \varphi} \leq c\left\{\|a\|_{\infty}\|f\|_{*, \varphi}+\|a\|_{*, \widetilde{\varphi}}\|f\|_{B M O_{\varphi}}\right\} .
$$

Note that $B M O_{\widetilde{\varphi}}(\Omega) \subseteq B M O_{\varphi}(\Omega)$; for instance, if $\varphi=1$, then $\widetilde{\varphi} \simeq$ $1 /|\log r|$ and we get Stegenga's result: $L M O \cap L^{\infty}$ multiplies $B M O$.

Proof. Following [33], p.582 we have

$$
\left|f_{B_{r}}\right| a f-(a f)_{B_{r}}\left|-\frac{\left|f_{B_{r}}\right|}{\left|B_{r}\right|} \int_{B_{r}}\right| a-a_{B_{r}}|| \leq 2 f_{B_{r}}|a|\left|f-f_{B_{r}}\right| .
$$

Dividing both members for $\varphi(r)$ and applying (2.2) we get

$$
\begin{aligned}
f\left|a f-(a f)_{B_{r}}\right| & \leq \frac{2\|a\|_{\infty}}{\varphi(r)} f_{B_{r}}\left|f-f_{B_{r}}\right|+\|f\|_{B M O_{\varphi}} \frac{1}{\widetilde{\varphi}(r)} f_{B_{r}}\left|a-a_{B_{r}}\right| \\
& \leq c\left\{\|a\|_{\infty}\|f\|_{*, \varphi}+\|a\|_{*, \widetilde{\varphi}}\|f\|_{B M O \varphi}\right\} .
\end{aligned}
$$

Lemma 2.5 (John-Nirenberg type) For any $f \in B M O_{\varphi}(\Omega), a \in B M O_{\widetilde{\varphi}}(\Omega)$ and $1 \leq p<\infty$,

$$
\begin{aligned}
& \frac{1}{\varphi(r)}\left(f_{B_{r}}\left|f-f_{B_{r}}\right|^{p}\right)^{1 / p} \leq c\|f\|_{*, \varphi} \\
& \frac{\left|f_{B_{r}}\right|}{\varphi(r)}\left(f_{B_{r}}\left|a-a_{B_{r}}\right|^{p}\right)^{1 / p} \leq c\|f\|_{B M O \varphi}\|a\|_{*, \widetilde{\varphi}} .
\end{aligned}
$$


Proof. The second inequality follows from the first and (2.2). The first follows from the standard John-Nirenberg inequality (see [8]) and monotonicity of $\varphi$. Namely, let $B$ denote any ball and $r(B)$ its radius, then:

$$
\begin{aligned}
\frac{1}{\varphi(r)}\left(f_{B_{r}}\left|f-f_{B_{r}}\right|^{p}\right)^{1 / p} & \leq \frac{1}{\varphi(r)} c \cdot \sup _{B \subseteq B_{r}} f_{B}\left|f-f_{B}\right| \\
& \leq c \cdot \sup _{B \subseteq B_{r}} \frac{1}{\varphi(r(B))} f_{B}\left|f-f_{B}\right| \leq c\|f\|_{*, \varphi} .
\end{aligned}
$$

Corollary 2.6 In $\mathrm{BMO}_{\varphi}(\Omega)$, with $\Omega$ bounded, all the norms

$$
\|f\|_{*, \varphi}+\|f\|_{p}(\text { for } 1 \leq p<\infty)
$$

are equivalent.

In the following we will often apply the multiplication theorem (Theorem 2.4) to a "smooth" function $a$. This is possible in view of the following fact: if $a$ is any smooth function and $\varphi$ satisfies the "logarithmic vanishing property" (1.4), then a belongs to $V M O_{\varphi}$. More generally:

Lemma 2.7 Let a be a Hölder continuous function on a space of homogeneous type $X$ :

$$
|a(x)-a(y)| \leq|a|_{C^{\alpha}} \cdot d(x, y)^{\alpha}
$$

for any $x, y \in X$, some $\alpha>0$. Then $a \in V M O_{\varphi}$, for every $\varphi$ satisfying the assumptions in $\S 1.2$. Therefore, a multiplies $\mathrm{BMO}_{\varphi}$ :

$$
\|a f\|_{*, \varphi} \leq c\left\{\|a\|_{\infty}\|f\|_{*, \varphi}+|a|_{C^{\alpha}}\|f\|_{B M O_{\varphi}}\right\} \text { for any } f \in B M O_{\varphi} .
$$

In the Euclidean case, for the standard $B M O$ space, the last estimate is known (see Lemma 3.2 in [36]).

Proof. By continuity of $a, a_{B_{r}}=a(\bar{x})$ for some $\bar{x} \in B_{r}$. Then

$$
\frac{1}{\varphi(r)} f_{B_{r}}\left|a-a_{B_{r}}\right| \leq c \frac{r^{\alpha}}{\varphi(r)} \rightarrow 0 \text { for } r \rightarrow 0
$$

by (1.4)

We end this section with some miscellaneous estimates, involving balls and "radial" functions, which hold in a general space of homogeneous type, and will be used over and over in the following. The techniques used in the proof of these estimates are standard, except for the presence of the function $\varphi$. 
Lemma 2.8 Let $X$ be any space of homogeneous type. Then

a. $\quad \int_{d(x, y)<r} \frac{d(x, y)^{\beta}}{|B(x ; y)|} d y \leq c r^{\beta} \quad$ for any $\beta>0 ;$
b. $\quad \int_{d(x, y)>r} \frac{d(x, y)^{-\beta}}{|B(x ; y)|} d y \leq c r^{-\beta}$ for any $\beta>0 ;$

for every locally integrable $f$

c. $\quad \frac{1}{\varphi(r)} \int_{d(x, y)<r} \frac{d(x, y)^{\beta}}{|B(x ; y)|}\left|f(y)-f_{B_{r}(x)}\right| d y \leq c r^{\beta}\|f\|_{*, \varphi}$ for any $\beta>0$;

d. $\quad \frac{1}{\varphi(r)} \int_{d(x, y)>r} \frac{1}{d(x, y)^{\beta}|B(x ; y)|}\left|f(y)-f_{B_{r}(x)}\right| d y \leq c r^{-\beta}\|f\|_{*, \varphi}$ for any $\beta>0$,

where all the constants are independent of $r>0$.

Proof. We will prove c, the other proofs being similar or easier.

$$
\begin{aligned}
& \frac{1}{\varphi(r)} \int_{d(x, y)<r} \frac{d(x, y)^{\beta}}{|B(x ; y)|}\left|f(y)-f_{B_{r}(x)}\right| d y \\
& =\frac{1}{\varphi(r)} \sum_{k=0}^{+\infty} \int_{2^{-k-1} r \leq d(x, y)<2^{-k} r} \frac{d(x, y)^{\beta}}{|B(x ; y)|}\left|f(y)-f_{B_{r}(x)}\right| d y \\
& \leq \frac{1}{\varphi(r)} \sum_{k=0}^{+\infty}\left(2^{-k} r\right)^{\beta} \frac{1}{\left|B\left(x, 2^{-k-1} r\right)\right|} \int_{B\left(x, 2^{-k} r\right)}\left|f(y)-f_{B_{r}(x)}\right| d y \\
& \leq \frac{c}{\varphi(r)} \sum_{k=0}^{+\infty}\left(2^{-k} r\right)^{\beta}\left(\frac{\varphi\left(2^{-k} r\right)}{\varphi\left(2^{-k} r\right)} f_{B\left(x, 2^{-k} r\right)}\left|f(y)-f_{B_{2^{-} k_{r}}}\right| d y+\left|f_{B_{2^{-k}}}-f_{B_{r}}\right|\right)
\end{aligned}
$$

(as in the proof of Lemma 2.2, and since $\varphi$ is nondecreasing)

$$
\begin{aligned}
& \leq c r^{\beta} \sum_{k=0}^{+\infty} 2^{-\beta k}\left(\frac{\varphi\left(2^{-k} r\right)}{\varphi(r)}+\sum_{j=0}^{k} \frac{\varphi\left(2^{-j} r\right)}{\varphi(r)}\right)\|f\|_{*, \varphi} \\
& \leq c r^{\beta} \sum_{k=0}^{+\infty} 2^{-\beta k}(2+k)\|f\|_{*, \varphi}=c r^{\beta}\|f\|_{*} .
\end{aligned}
$$




\section{Continuity of singular and fractional integral opera- tors on $\mathrm{BMO}_{\varphi}$}

Definition 3.1 Let $(X, d, d x)$ be a space of homogeneous type.

We will say that a measurable function $k(x, y): X \times X \rightarrow \mathbb{R}$ is a standard kernel on $X$ if $k$ satisfies the following properties:

$$
|k(x, y)| \leq \frac{c}{|B(x ; y)|} \quad \text { for any } x, y \in X
$$

("growth estimate")

$$
\left|k(x, y)-k\left(x_{0}, y\right)\right| \leq \frac{c}{\left|B\left(x_{0} ; y\right)\right|}\left(\frac{d\left(x_{0}, x\right)}{d\left(x_{0}, y\right)}\right)^{\beta}
$$

for any $x_{0}, x, y \in X$, with $d\left(x_{0}, y\right) \geq M d\left(x_{0}, x\right), M>1, c, \beta>0$ ("mean value inequality").

We will say that $K$ is a Calderón-Zygmund operator on $X$ if:

1. $K$ is continuous on $L^{p}(X)$ for $1<p<\infty$;

2. there exists a standard kernel $k(x, y)$ on $X$ such that for every $f \in L^{p}$, we have:

$$
K f(x)=P . V . \int_{X} k(x, y) f(y) d y .
$$

Remark 3.2 If condition (3.2) holds for some $M_{0}>1$, then it holds for any $M \geq M_{0}$. We can assume $M$ large enough, so that the condition $d\left(x_{0}, y\right) \geq$ $M d\left(x_{0}, x\right)$ implies that $d\left(x_{0}, y\right) \simeq d(x, y)$. We will use systematically this equivalence. Also, just not to use one more constant, we will assume that this "large" value of $M$ is 2 . This means to assume that the constant $c_{d}$ in $(1.2)$ is $<2$. The reader will excuse this little abuse of notation.

Let $K$ be a Calderón-Zygmund operator on a space of homogeneous type. Then, it is easy to prove (see below) that

$$
K: L^{\infty}(X) \rightarrow B M O(X) .
$$

For classical Calderón-Zygmund operators, (3.3) is a well-known result (see for instance [34], pp. 155-157). It is much less easy to prove that

$$
K: B M O(X) \rightarrow B M O(X)
$$

or

$$
K: B M O_{\varphi}(X) \rightarrow B M O_{\varphi}(X) .
$$


In the classical case, (3.4) holds on $X=\mathbb{R}^{n}$, provided $K$ is of convolution type (see Peetre [27]). We will prove (3.4) for any Calderón-Zygmund operator on a space of homogeneous type of finite measure, provided the kernel satisfies a suitable cancellation property.

Lemma 3.3 Let us define the functions $\eta, \psi:[0,+\infty) \rightarrow \mathbb{R}$ :

$$
\begin{gathered}
\eta(t)= \begin{cases}1 & \text { for } 0 \leq t \leq 1 \\
2-t & \text { for } 1 \leq t \leq 2 \\
0 & \text { for } t \geq 2\end{cases} \\
\psi(t)=\eta(t)-\eta(2 t)
\end{gathered}
$$

set:

$$
\psi_{j}(x, y)=\psi\left(2^{-j} d(x, y)\right)=\eta\left(2^{-j} d(x, y)\right)-\eta\left(2^{-j+1} d(x, y)\right) .
$$

Then:

$$
\begin{gathered}
\sum_{j=-\infty}^{+\infty} \psi_{j}(x, y)= \begin{cases}1 & \text { for } x \neq y \\
0 & \text { for } x=y ;\end{cases} \\
\left|\psi_{j}(x, y)-\psi_{j}(z, y)\right| \leq 2^{-j+1}|d(x, y)-d(z, y)| \\
\psi_{j}(x, y) \neq 0 \text { only if } 2^{j-1}<d(x, y)<2^{j+1} .
\end{gathered}
$$

The proof is immediate from the definitions.

To prove the $\mathrm{BMO}_{\varphi}$ continuity of $K$ on spaces of finite measure, we proceed as follows. Let

$$
k_{j}(x, y)=k(x, y) \psi_{j}(x, y)
$$

with $\psi_{j}(x, y)$ as in Lemma 3.3, and

$$
K_{j} f(x)=\int_{X} k_{j}(x, y) f(y) d y
$$

so that

$$
\begin{aligned}
K f(x) & =P . V \cdot \int_{X} k(x, y) f(y) d y \\
& =\sum_{j=-\infty}^{+\infty} \int_{X} k_{j}(x, y) f(y) d y=\sum_{j=-\infty}^{+\infty} K_{j} f(x)
\end{aligned}
$$


Theorem 3.4 Let $K$ be a Calderón-Zygmund operator on a space of homogeneous type $(X, d, d x)$. Then

$$
\|K f\|_{*} \leq c\|f\|_{\infty} .
$$

Moreover, assume that $X$ has finite measure and the kernel of $K$ satisfies the strong vanishing property:

$$
\int_{X} k_{i}(x, z) d z=0 \text { for every } x \in X, i \in \mathbb{Z}
$$

with $k_{i}$ as above. Then

$$
\|K f\|_{*, \varphi} \leq c\|f\|_{*, \varphi} .
$$

Proof. First of all we observe that:

$$
f_{B_{r}\left(x_{0}\right)}\left|f(x)-f_{B_{r}}\right| d x \leq 2 f_{B_{r}\left(x_{0}\right)}|f(x)-\tau| d x .
$$

Therefore, to control a $B M O_{\varphi}$ norm it is enough to control the integral in the RHS with a suitable $\tau$. We first prove (3.9). For any ball $B_{r}\left(x_{0}\right)$ we can estimate:

$$
f_{B_{r}\left(x_{0}\right)}|K f(x)-\tau| d x \leq
$$

(choosing $\tau=\int_{B_{2 r}^{c}\left(x_{0}\right)} k\left(x_{0}, y\right) f(y) d y$ (where $B_{r}^{c}$ denotes the complement of $\left.B_{r}\right)$ )

$\leq f_{B_{r}}\left|K\left(f \chi_{B_{2 r}}\right)(x)\right| d x+f_{B_{r}} \int_{B_{2 r}^{c}}\left|k(x, y)-k\left(x_{0}, y\right)\right||f(y)| d y d x=I+I I$.

Then, by Hölder, $L^{p}$ continuity of $K$ and the doubling condition:

$$
I \leq\left(f_{B_{r}}\left|K\left(f \chi_{B_{2 r}}\right)(x)\right|^{p} d x\right)^{1 / p} \leq c\left(f_{B_{2 r}}|f(x)|^{p} d x\right)^{1 / p} \leq c\|f\|_{\infty}
$$

while

$$
I I \leq c\|f\|_{\infty} f_{B_{r}} d\left(x_{0}, x\right)^{\alpha} d x \int_{B_{2 r}^{c}} \frac{1}{\left|B\left(x_{0} ; y\right)\right| d\left(x_{0}, y\right)^{\alpha}} d y \leq c\|f\|_{\infty}
$$

by (3.2) and Lemma 2.8b. This proves (3.9).

Proof of (3.11). Since, by assumption, $K$ is bounded on $L^{p}$, and the series in (3.8) converges, for any $p \in(1, \infty), f \in L^{p}$,

$$
\sup _{k_{1}, k_{2}}\left\|\sum_{j=-k_{1}}^{k_{2}} K_{j} f\right\|_{p}<\infty
$$


Then, Banach-Steinhaus' theorem implies the uniform estimate:

$$
\left\|\sum_{j=-k_{1}}^{k_{2}} K_{j} f\right\|_{p} \leq c\|f\|_{p}
$$

for any $p \in(1, \infty)$, for some $c$ independent of $f$ and $k_{1}, k_{2}>0$, any $f \in L^{p}$.

Recall that

$$
k_{j}(x, y) \neq 0 \text { only if } 2^{j-1}<d(x, y)<2^{j+1} .
$$

Let us write:

$$
\begin{aligned}
\frac{1}{\varphi(r)} f_{B_{r}\left(x_{0}\right)}\left|\sum_{-\infty}^{+\infty} K_{j} f(x)-\tau\right| d x \leq & \frac{1}{\varphi(r)}\left(f_{B_{r}\left(x_{0}\right)}\left|\sum_{2^{j-2} \leq r} K_{j} f(x)\right|^{p} d x\right)^{1 / p} \\
& +\frac{1}{\varphi(r)} f_{B_{r}\left(x_{0}\right)}\left|\sum_{2^{j-2}>r} K_{j} f(x)-\tau\right| d x .
\end{aligned}
$$

We will bound the two terms separately. Consider first the case $2^{j-2} \leq r$. Then:

$$
\sum_{j=-k_{1}}^{k_{2}} K_{j} f(x)=\sum_{j=-k_{1}}^{k_{2}} \int_{X} k_{j}(x, y) f(y) d y=
$$

(by $(3.10)$ )

$$
\begin{aligned}
= & \int_{X} \sum_{j=-k_{1}}^{k_{2}} k_{j}(x, y)[f(y)-\sigma] d y= \\
& \left(\text { if } x \in B_{r}\left(x_{0}\right), d(x, y)<2^{j+1} \leq 8 r ; d\left(x_{0}, y\right) \leq c_{d}(r+8 r)=9 c_{d} r\right) \\
= & \int_{B_{c r}\left(x_{0}\right)} \sum_{j=-k_{1}}^{k_{2}} k_{j}(x, y)[f(y)-\sigma] d y=\sum_{j=-k_{1}}^{k_{2}} K_{j}\left([f(y)-\sigma] \chi_{B_{c r}\left(x_{0}\right)}\right)(x)
\end{aligned}
$$

so that, by (3.13):

$$
\int_{B_{r}\left(x_{0}\right)}\left|\sum_{j=-k_{1}}^{k_{2}} K_{j} f(x)\right|^{p} d x \leq c \int_{B_{c r}\left(x_{0}\right)}|f(y)-\sigma|^{p} d y
$$

and, choosing $\sigma=f_{B_{c r}\left(x_{0}\right)}$

$$
\frac{1}{\varphi(r)}\left(f_{B_{r}\left(x_{0}\right)}\left|\sum_{j: 2^{j-2} \leq r} K_{j} f(x)\right|^{p} d x\right)^{1 / p} \leq c\|f\|_{*, \varphi}
$$

by Lemma 2.5 and since $\varphi$ is doubling. 
Let now $2^{j-2}>r$. Then

$$
K_{j} f(x)-K_{j} f\left(x_{0}\right)=\int_{X}\left[k_{j}(x, y)-k_{j}\left(x_{0}, y\right)\right]\left[f(y)-\sigma_{j}\right] d y
$$

with $\sigma_{j}$ to be chosen later. Let $\tau=\sum_{j: 2^{j-2}>r} K_{j} f\left(x_{0}\right)$, then

$$
\begin{aligned}
\frac{1}{\varphi(r)} & f_{B_{r}\left(x_{0}\right)}\left|\sum_{j: 2^{j-2}>r} K_{j} f(x)-\tau\right| d x \\
\leq & \frac{1}{\varphi(r)} f_{B_{r}\left(x_{0}\right)} d x \int\left|k(x, y)-k\left(x_{0}, y\right)\right| \sum_{j} \psi_{j}(x, y)\left|f(y)-\sigma_{j}\right| d y \\
& +\frac{1}{\varphi(r)} f_{B_{r}\left(x_{0}\right)} d x \int \sum_{j}\left|\psi_{j}(x, y)-\psi_{j}\left(x_{0}, y\right)\right|\left|k\left(x_{0}, y\right)\right|\left|f(y)-\sigma_{j}\right| d y \\
= & A+B .
\end{aligned}
$$

To bound $A$, we note that since $\psi_{j}(x, y) \neq 0$ only if $d(x, y) \geq 2^{j-1}$, we have:

$$
d(x, y) \geq 2^{j-1} \geq 2 r \geq 2 d\left(x, x_{0}\right),
$$

therefore we can apply the mean value inequality (3.2); moreover, since $d(x, y) \geq 2^{j-1}$ implies $d\left(x_{0}, y\right) \geq c 2^{j-1}$ we can write

$$
\begin{aligned}
A \leq & \frac{c}{\varphi(r)} f_{B_{r}\left(x_{0}\right)} d\left(x, x_{0}\right)^{\alpha} d x \sum_{j: 2^{j-2}>r} \frac{\varphi\left(c 2^{j-1}\right)}{\varphi\left(c 2^{j-1}\right)} \int_{d\left(x_{0}, y\right) \geq c 2^{j-1}} \frac{\left|f(y)-\sigma_{j}\right|}{d\left(x_{0}, y\right)^{\alpha}\left|B\left(x_{0} ; y\right)\right|} d y \\
& \left(\operatorname{choosing} \sigma_{j}=f_{B_{c 2^{j-1}}},\right. \text { by Lemma 2.8d) } \\
\leq & \frac{c r^{\alpha}}{\varphi(r)} \sum_{j: 2^{j-2}>r} \varphi\left(c 2^{j-1}\right) 2^{-j \alpha}\|f\|_{*, \varphi} .
\end{aligned}
$$

We now exploit assumption (1.5) on $\varphi$. Let $j_{0}=\left[\log _{2} 4 r\right]$; then

$$
\begin{aligned}
\varphi\left(c 2^{j-1}\right) & \leq c \varphi\left(2^{j}\right)=c \varphi\left(2^{j-j_{0}} \cdot 2^{j_{0}}\right) \\
& \leq c\left(1+j-j_{0}\right)^{\beta} \varphi\left(2^{j_{0}}\right) \leq c\left(1+j-j_{0}\right)^{\beta} \varphi(4 r) .
\end{aligned}
$$

Hence

$$
\begin{aligned}
A & \leq c r^{\alpha} \frac{\varphi(4 r)}{\varphi(r)}\|f\|_{*, \varphi} \sum_{j=j_{0}}^{\infty} 2^{-j \alpha} c\left(1+j-j_{0}\right)^{\beta} \leq \\
& \leq c r^{\alpha} \sum_{j=0}^{\infty}(1+j)^{\beta} 2^{-j \alpha} \cdot 2^{-j_{0} \alpha}\|f\|_{*, \varphi} \leq c r^{\alpha} \cdot r^{-\alpha}\|f\|_{*, \varphi}=c\|f\|_{*, \varphi} .
\end{aligned}
$$


To bound $B$, recall that, by (3.6) and Theorem 1.3,

$$
\begin{aligned}
\left|\psi_{j}(x, y)-\psi_{j}\left(x_{0}, y\right)\right| & \leq 2^{-j+1}\left|d(x, y)-d\left(x_{0}, y\right)\right| \\
& \leq c 2^{-j} d\left(x_{0}, x\right)^{\alpha}\left[d\left(x_{0}, y\right)^{1-\alpha}+d(x, y)^{1-\alpha}\right] .
\end{aligned}
$$

We claim that the previous inequality can be improved as follows:

$$
\left|\psi_{j}(x, y)-\psi_{j}\left(x_{0}, y\right)\right| \leq c 2^{-j} d\left(x_{0}, x\right)^{\alpha} d\left(x_{0}, y\right)^{1-\alpha} .
$$

Namely: if $d\left(x_{0}, y\right)<2^{j-1}$ and $d(x, y)<2^{j-1}$, then the left hand side vanishes. Otherwise, since $d\left(x_{0}, x\right)<r<2^{j-2}$ we can write

$$
d(x, y) \leq c_{d}\left(2^{j-2}+d\left(x_{0}, y\right)\right) .
$$

Now, if $2^{j-1} \leq d\left(x_{0}, y\right)$, the last inequality implies $d(x, y) \leq c d\left(x_{0}, y\right)$, and the claim is proved, while if $2^{j-1} \leq d(x, y)$ the last inequality implies

$$
d(x, y) \leq \frac{c_{d}}{2} d(x, y)+c_{d} d\left(x_{0}, y\right)
$$

and recalling that $c_{d}<2$ (see Remark 3.2), this again means that $d(x, y) \leq$ $c d\left(x_{0}, y\right)$.

From (3.14) we have:

$B \leq \frac{c}{\varphi(r)} \sum_{j: 2^{j-2}>r} 2^{-j} f_{B_{r}\left(x_{0}\right)} d\left(x, x_{0}\right)^{\alpha} d x \int_{d\left(x_{0}, y\right) \leq c 2^{j+1}} d\left(x_{0}, y\right)^{1-\alpha} \frac{\left|f(y)-f_{B_{c 2^{j}-1}}\right|}{\left|B\left(x_{0}, y\right)\right|} d y$

(by Lemma 2.8c)

$$
\begin{aligned}
& \leq \frac{c}{\varphi(r)} \sum_{j=j_{0}}^{\infty} 2^{-j} r^{\alpha} \varphi\left(c 2^{j+1}\right)\left(c 2^{j+1}\right)^{1-\alpha}\|f\|_{*, \varphi} \\
& \leq \frac{c r^{\alpha}}{\varphi(r)} \sum_{j=j_{0}}^{\infty} 2^{-\alpha j} \varphi\left(2^{j}\right)\|f\|_{*, \varphi} \leq c\|f\|_{*, \varphi}
\end{aligned}
$$

where in the last inequality we have exploited (1.4) as in the estimate of $A$. Finally,

$$
\begin{aligned}
& \frac{1}{\varphi(r)} f_{B_{r}\left(x_{0}\right)}\left|\sum_{-\infty}^{+\infty} K_{j} f(x)-\tau\right| d x \\
& \leq \frac{1}{\varphi(r)}\left(f_{B_{r}\left(x_{0}\right)}\left|\sum_{2^{j-2} \leq r} K_{j} f(x)\right|^{p} d x\right)^{1 / p}+\frac{1}{\varphi(r)} f_{B_{r}\left(x_{0}\right)}\left|\sum_{2^{j-2}>r} K_{j} f(x)-\tau\right| d x \\
& \leq c\|f\|_{*, \varphi} .
\end{aligned}
$$

We will need also a similar result for fractional integral operators on spaces of homogeneous type. 
Theorem 3.5 Let $X$ be a space of homogeneous type of finite measure, and let $k_{\alpha}(x, y)$ (for any $\alpha \in(0,1)$ ) be a "fractional integral kernel", such that:

$$
\begin{aligned}
\left|k_{\alpha}(x, y)\right| & \leq \frac{c}{|B(x ; y)|^{1-\alpha}} \\
\left|k_{\alpha}(x, y)-k_{\alpha}\left(x_{0}, y\right)\right| & \leq \frac{c}{\left|B\left(x_{0} ; y\right)\right|^{1-\alpha}}\left(\frac{d\left(x_{0}, x\right)}{d\left(x_{0}, y\right)}\right)^{\beta}
\end{aligned}
$$

for some positive constants $c, \beta$, any $x_{0}, x, y$ such that $d\left(x_{0}, y\right) \geq 2 d\left(x_{0}, x\right)$. Assume that the space satisfies the following geometric property (a kind of "reverse doubling condition"):

$$
\frac{\left|B_{r}(x)\right|}{\left|B_{r_{0}}(x)\right|} \leq c\left(\frac{r}{r_{0}}\right)^{\gamma}
$$

for any $r, r_{0}$ with $r<r_{0}<R$, some positive $c, \gamma$. Let

$$
I_{\alpha} f(x)=\int k_{\alpha}(x, y) f(y) d y
$$

Then

$$
\left\|I_{\alpha} f\right\|_{B M O_{\varphi}} \leq c\|f\|_{B M O_{\varphi}}
$$

Remark 3.6 a. For the standard BMO space (that is, $\varphi=1$ ), from the proof below, the following estimate can be obtained:

$$
\left\|I_{\alpha} f\right\|_{*} \leq c\|f\|_{L^{1 / \alpha}} .
$$

This theorem is well known in Euclidean spaces (see for instance 6.29 p. 221 in [35]).

b. Note that, in a general space of homogeneous type, property (3.16) does not hold automatically for the "natural" kernel $k_{\alpha}(x, y)=|B(x ; y)|^{\alpha-1}$, but has to be required axiomatically. (See [3] for a discussion of this fact and how this problem can be sometimes bypassed).

c. Condition (3.17) holds, for instance, if $(X, d, d x)$ is a space with no "atoms", which satisfies "condition P" stated in [3], [7]. Assumption (3.17) could be weakened, but here we are not interested in stating these results in their full generality. 
530 M. Bramanti and L. Brandolini

Proof.

$$
\begin{aligned}
& \frac{1}{\varphi(r)} f_{B_{r}\left(x_{0}\right)}\left|I_{\alpha} f(x)-\tau\right| d x \\
& \leq \frac{1}{\varphi(r)} f_{B_{r}\left(x_{0}\right)} \int_{d\left(x_{0}, y\right)<2 r}\left|k_{\alpha}(x, y) f(y)\right| d y \\
& \quad+\int_{d\left(x_{0}, y\right) \geq 2 r}\left|\left[k_{\alpha}(x, y)-k_{\alpha}\left(x_{0}, y\right)\right] f(y)\right| d y d x \\
& \quad \equiv A+B,
\end{aligned}
$$

having chosen

$$
\tau=\int_{d\left(x_{0}, y\right) \geq 2 r} k_{\alpha}\left(x_{0}, y\right) f(y) d y
$$

Now,

$$
\begin{aligned}
A & \leq \frac{1}{\varphi(r)} f_{B_{r}\left(x_{0}\right)} \int_{d(x, y)<c r} \frac{|f(y)|}{|B(x ; y)|^{1-\alpha}} d y d x \\
& \leq \frac{c}{\varphi(r)} f_{B_{r}\left(x_{0}\right)} \sum_{k=0}^{\infty} \int_{\frac{c r}{2^{k+1}} \leq d(x, y)<\frac{c r}{2^{k}}} \frac{|f(y)|}{|B(x ; y)|^{1-\alpha}} d y d x \\
\leq & \frac{c}{\varphi(r)} f_{B_{r}\left(x_{0}\right)} \sum_{k=0}^{\infty}\left|B_{\frac{c r}{2^{k+1}}}(x)\right|^{\alpha}\left(f_{B_{\frac{c r}{2^{k}}}(x)}\left|f(y)-f_{B_{\frac{c r}{2^{k}}}}\right| d y+\left|f_{B_{\frac{c r}{2^{k}}}}\right|\right) d x \\
\leq & \quad f_{B_{r}\left(x_{0}\right)} \sum_{k=0}^{\infty}\left|B_{\frac{c r}{2^{k+1}}}(x)\right|^{\alpha} \frac{\varphi\left(\frac{c r}{2^{k}}\right)}{\varphi(r)}\|f\|_{*, \varphi} d x+ \\
& +c f_{B_{r}\left(x_{0}\right)} \sum_{k=0}^{\infty}\left|B_{\frac{c r}{2^{k+1}}}(x)\right|^{\alpha} \frac{\left|f_{B_{c r 2}-k}\right|}{\varphi(r)} d x \\
= & C_{1}+C_{2} .
\end{aligned}
$$

Since $\varphi\left(\frac{c r}{2^{k}}\right) / \varphi(r) \leq c$, by $(3.17)$

$$
C_{1} \leq c\|f\|_{*, \varphi} f_{B_{r}\left(x_{0}\right)}\left|B_{r}(x)\right|^{\alpha} d x \leq c r^{\gamma}\|f\|_{*, \varphi}
$$

for some positive $\gamma$. By Lemmas 2.2 and 2.3,

$$
\left|f_{B_{c r 2^{-k}}}\right| \leq c\left(\|f\|_{1}+\|f\|_{*, \varphi} S_{\varphi}\left(\frac{r}{2^{k}}\right)\right) .
$$

Since

$$
S_{\varphi}\left(\frac{r}{2^{k}}\right)=\int_{r / 2^{k}}^{2 R} \frac{\varphi(t)}{t} d t \leq \varphi(2 R)\left[\log \left(\frac{2 R}{r}\right)+k \log 2\right]
$$


we can write

$$
\begin{aligned}
C_{2} \leq & c\|f\|_{1} f_{B_{r}\left(x_{0}\right)} \frac{r^{\gamma}}{\varphi(r)} d x \\
& +c\|f\|_{*, \varphi} \frac{\varphi(2 R)}{\varphi(r)} f_{B_{r}\left(x_{0}\right)}\left[\log \left(\frac{2 R}{r}\right) r^{\gamma}+\left|B_{r}(x)\right|^{\alpha} \sum_{k=0}^{\infty} \frac{k}{2^{k \gamma}}\right] d x \\
\leq & \frac{c r^{\gamma}}{\varphi(r)}\|f\|_{1}+\frac{c r^{\gamma}}{\varphi(r)} \varphi(2 R)\left[\log \left(\frac{2 R}{r}\right)+1\right]\|f\|_{*, \varphi} .
\end{aligned}
$$

By (1.4), $\frac{r^{\gamma}}{\varphi(r)}$ and $\frac{r^{\gamma}}{\varphi(r)} \varphi(2 R) \log \left(\frac{2 R}{r}\right)$ vanish with $r$. Hence we have proved that

$$
A \leq \omega(r)\|f\|_{*, \varphi}
$$

with $\omega(r) \rightarrow 0$ for $r \rightarrow 0$.

To bound $B$ :

$B \leq \frac{c}{\varphi(r)} f_{B_{r}\left(x_{0}\right)} d\left(x_{0}, x\right)^{\beta} d x \int_{d\left(x_{0}, y\right) \geq 2 r} \frac{\left|f(y)-f_{B_{2 r}}\right|+\left|f_{B_{2 r}}\right|}{\left|B\left(x_{0} ; y\right)\right|^{1-\alpha} d\left(x_{0}, y\right)^{\beta}} d y=D_{1}+D_{2}$.

By Lemma 2.8d,

$$
D_{1} \leq c f_{B_{r}\left(x_{0}\right)} d\left(x_{0}, x\right)^{\beta} d x|\Omega|^{\alpha}\|f\|_{*, \varphi} r^{-\beta} \leq c|\Omega|^{\alpha}\|f\|_{*, \varphi} ;
$$

by $(2.2)$,

$$
\begin{aligned}
D_{2} \leq & c\|f\|_{B M O_{\varphi}} \frac{1}{\widetilde{\varphi}(r)} f_{B_{r}\left(x_{0}\right)} d\left(x_{0}, x\right)^{\beta} d x \int_{d\left(x_{0}, y\right) \geq 2 r} \frac{1}{\left|B\left(x_{0} ; y\right)\right|^{1-\alpha} d\left(x_{0}, y\right)^{\beta}} d y \\
& \left(\text { with } 2^{k} \simeq R / 2 r\right) \\
& \leq c\|f\|_{B M O_{\varphi}} \frac{r^{\beta}}{\widetilde{\varphi}(r)} \sum_{j=1}^{k} \int_{\frac{R}{2^{j}} \leq d\left(x_{0}, y\right)<\frac{R}{2^{j-1}}} \frac{1}{\left|B\left(x_{0} ; y\right)\right|^{1-\alpha} d\left(x_{0}, y\right)^{\beta}} d y \\
& \leq c\|f\|_{B M O_{\varphi}} \frac{r^{\beta}}{\widetilde{\varphi}(r)} \sum_{j=1}^{k} \frac{\left|B\left(x_{0}, \frac{R}{2^{j}}\right)\right|^{\alpha}}{\left(\frac{R}{2^{j}}\right)^{\beta}} .
\end{aligned}
$$

To conclude the proof, we have to show that the function

$$
\frac{r^{\beta}}{\widetilde{\varphi}(r)} \sum_{j=1}^{k} \frac{\left|B\left(x_{0}, \frac{R}{2^{j}}\right)\right|^{\alpha}}{\left(\frac{R}{2^{j}}\right)^{\beta}}
$$

is bounded for small $r$ (actually, we will show that it vanishes with $r$ ). 
Since, by Lemma 2.1, the function $\widetilde{\varphi}$ satisfies the "logarithmic vanishing property" (1.4), it will be enough to show that the function

$$
g(r) \equiv r^{\beta} \sum_{j=1}^{k} \frac{\left|B\left(x_{0}, \frac{R}{2^{j}}\right)\right|^{\alpha}}{\left(\frac{R}{2^{j}}\right)^{\beta}}
$$

vanishes like some positive power of $r$, for $r \rightarrow 0$. To see this, we exploit (3.17):

$$
g(r) \leq r^{\beta} \frac{\left|B\left(x_{0}, R\right)\right|^{\alpha}}{R^{\beta}} \sum_{j=1}^{k} 2^{j(\beta-\alpha \gamma)} .
$$

We now distinguish the three cases:

i. $\beta-\alpha \gamma \equiv-\delta<0$. Then

$$
g(r) \leq c r^{\beta} \frac{\left|B\left(x_{0}, R\right)\right|^{\alpha}}{R^{\beta}} \leq c r^{\beta}
$$

ii. $\beta-\alpha \gamma \equiv \delta>0$. Then, recalling that $2^{k} \simeq \frac{R}{2 r}$

$$
\begin{aligned}
g(r) & \leq r^{\beta} \frac{\left|B\left(x_{0}, R\right)\right|^{\alpha}}{R^{\beta}} k 2^{k \delta} \leq c r^{\beta} \frac{\left|B\left(x_{0}, R\right)\right|^{\alpha}}{R^{\beta}}\left(\frac{R}{r}\right)^{\beta-\alpha \gamma} \log _{2} \frac{R}{r} \\
& \leq c r^{\alpha \gamma} \frac{\left|B\left(x_{0}, R\right)\right|^{\alpha}}{R^{\alpha \gamma}} \log _{2} \frac{R}{r} \leq c r^{\alpha \gamma-\varepsilon}
\end{aligned}
$$

for any $\varepsilon<\alpha \gamma$, and small $r$.

iii. $\beta-\alpha \gamma=0$. Then

$$
g(r) \leq r^{\beta} \frac{\left|B\left(x_{0}, R\right)\right|^{\alpha}}{R^{\beta}} k \leq c r^{\beta} \frac{\left|B\left(x_{0}, R\right)\right|^{\alpha}}{R^{\beta}} \log _{2} \frac{R}{r} \leq c r^{\beta-\varepsilon}
$$

for any $\varepsilon<\beta$, and small $r$.

Therefore

$$
D_{2} \leq c\|f\|_{B M O_{\varphi}}
$$

for small $r$. This finishes the proof. 


\section{Applications to uniformly hypoelliptic operators}

\subsection{Some geometric preliminaries}

Let $\Omega$ be a bounded domain of $\mathbb{R}^{n}$ (throughout this section the word "bounded" always refers to the Euclidean metric), and let $X_{1}, X_{2}, \ldots, X_{q}$ $(q<n)$ be a system of smooth real vector fields defined in a neighborhood $\Omega_{o}$ of $\Omega$ and satisfying Hörmander's condition of step $s$ in $\Omega_{o}$. Let $d$ be the Carnot-Carathéodory distance induced in $\Omega$ by this system (see for instance [26] for the definition of $d$ ). For $x \in \Omega$, let $B_{r}(x)=\left\{y \in \Omega_{o}: d(x, y)<r\right\}$. It is well known (see [26]) that there exist positive constants $c, r_{0}, c_{1}, c_{2}$ depending on $\Omega$ such that:

$$
\begin{gathered}
\left|B_{2 r}(x)\right| \leq c\left|B_{r}(x)\right| \text { for any } x \in \Omega, r \leq r_{0} \\
c_{1}|x-y| \leq d(x, y) \leq c_{2}|x-y|^{1 / s} \text { for any } x, y \in \Omega .
\end{gathered}
$$

In order to apply to this context the abstract theory of spaces of homogenous type developed in $\S \S 2-3$, we need to know that in $(\Omega, d, d x)$ the doubling condition holds. Explicitly, this means that

$$
\left|B_{2 r}(x) \cap \Omega\right| \leq c\left|B_{r}(x) \cap \Omega\right| \text { for any } x \in \Omega, r>0 .
$$

This requires some regularity property of $\partial \Omega$.

Definition 4.1 Under the above assumptions, we say that $\Omega$ is d-regular if

$$
\left|B_{r}(x) \cap \Omega\right| \geq c\left|B_{r}(x)\right|
$$

for every $x \in \Omega, 0<r<\operatorname{diam}(\Omega)$.

If $d$ is the Euclidean distance, the above property holds, for instance, if $\Omega$ is Lipschitz, or satisfies a uniform inner cone condition (i.e. outer cusps are not allowed); if $d$ is the Carnot-Carathéodory distance induced by a system of Hörmander's vector fields, then the metric balls can actually have cusps (from the Euclidean point of view); nevertheless, they turn out to be d-regular:

\section{Lemma 4.2}

i. Let $\Omega=B_{R}\left(x_{0}\right) \subset \Omega_{o}$ be a metric ball. Then, $B_{R}\left(x_{0}\right)$ is d-regular.

ii. The union of a finite number of d-regular domains in $\Omega_{o}$ is d-regular.

iii. If $\Omega$ is a bounded $d$-regular domain in $\Omega_{o}$, then $(\Omega, d, d x)$ is a space of homogeneous type. 


\section{Proof.}

i. First of all, note that (4.1) holds for any $r$. Indeed, for $r \geq r_{0}$

$$
\frac{\left|B_{2 r}(x)\right|}{\left|B_{r}(x)\right|} \leq \frac{\left|\Omega_{o}\right|}{\left|B_{r_{0}}(x)\right|} \leq c
$$

because, by (4.2), $\inf _{x \in \Omega}\left|B_{r_{0}}(x)\right| \geq c>0$.

Let $B_{R}\left(x_{0}\right)$ be a metric ball, $x \in B_{R}\left(x_{0}\right), d\left(x, x_{0}\right)=\rho<R$. Assume $r<3 \rho$ and let $\gamma:[0,1] \rightarrow B_{R}\left(x_{0}\right)$ be a subunit curve, connecting $x_{0}$ to $x$, of total length $\ell(\gamma)<\rho+\frac{r}{3}$. Let $x_{1}$ be a point of $\gamma$ such that the arc of $\gamma$ connecting $x_{0}$ to $x_{1}$ has length $\rho-\frac{r}{3}$ (and therefore the remaining part of $\gamma$ that connects $x_{1}$ to $x$ has length strictly less than $\frac{2}{3} r$ ). It follows that:

$$
\begin{aligned}
d\left(x_{0}, x_{1}\right) & <\rho-\frac{r}{3} \\
d\left(x_{1}, x\right) & <\frac{2}{3} r .
\end{aligned}
$$

Then

$$
B_{r / 3}\left(x_{1}\right) \subset B_{R}\left(x_{0}\right) \cap B_{r}(x) .
$$

Namely, for $z \in B_{r / 3}\left(x_{1}\right)$ we have:

$$
\begin{gathered}
d\left(z, x_{0}\right) \leq d\left(x_{1}, x_{0}\right)+d\left(x_{1}, z\right)<\rho-\frac{r}{3}+\frac{r}{3}=\rho<R \\
d(z, x)<d\left(z, x_{1}\right)+d\left(x_{1}, x\right)<\frac{r}{3}+\frac{2}{3} r=r .
\end{gathered}
$$

But then, by (4.1),

$$
\left|B_{R}\left(x_{0}\right) \cap B_{r}(x)\right| \geq\left|B_{r / 3}\left(x_{1}\right)\right| \geq c\left|B_{2 r}\left(x_{1}\right)\right| \geq c\left|B_{r}(x)\right| .
$$

If $r \geq 3 \rho$ the previous argument works taking $x_{1}=x_{0}$.

ii. Let $\Omega_{1}, \Omega_{2}$ be two $d$-regular domains, and pick $x \in \Omega_{1} \cup \Omega_{2}$. Then, assuming for instance that $x \in \Omega_{1}$,

$$
\left|B_{r}(x) \cap\left(\Omega_{1} \cup \Omega_{2}\right)\right| \geq\left|B_{r}(x) \cap \Omega_{1}\right| \geq c\left|B_{r}(x)\right|,
$$

because $\Omega_{1}$ is $d$-regular. The same reasoning holds for $n$ sets.

iii. Let $x \in \Omega$, then:

$$
\left|B_{r}(x) \cap \Omega\right| \geq c\left|B_{r}(x)\right| \geq c\left|B_{2 r}(x)\right| \geq c\left|B_{2 r}(x) \cap \Omega\right|
$$

where the first inequality holds because $\Omega$ is $d$-regular, the second by (4.1). 
Remark 4.3 In the proof of the previous Lemma we used only the definition of Carnot-Carathéodory distance as the infimum of the length of paths connecting two points, without requiring the existence of a minimizing geodesic, that can be guaranteed only under stronger assumptions. See e.g. [2] and the discussion on pp. 1086-7 of [19].

If $\Omega$ is $d$-regular, the space $B M O_{\varphi}(\Omega)$ is defined, explicitly, by the seminorm:

$$
\|f\|_{*, \varphi}=\sup _{x \in \Omega, r>0} \frac{1}{\left|B_{r}(x) \cap \Omega\right| \varphi(r)} \int_{B_{r} \cap \Omega}\left|f(y)-f_{B_{r} \cap \Omega}\right| d y .
$$

When $\Omega$ is implicitly understood, we will simply write $B_{r}(x)$ for

$$
\{y \in \Omega: d(x, y)<r\} .
$$

In this context, we will need several basic facts relating $B M O_{\varphi}$ spaces and norms relative to different domains of $\mathbb{R}^{n}$. These facts are contained in the following important lemma:

Lemma 4.4 Let us consider the space of homogeneous type $(\Omega, d, d x)$, where $\Omega$ is a d-regular, bounded domain of $\mathbb{R}^{n}$.

i. Let $\Omega^{\prime}$ be a d-regular subset of $\Omega$ such that, for some $\rho>0$ and $x_{0} \in \Omega$,

$$
\Omega^{\prime} \subset B_{\rho}\left(x_{0}\right) \subset B_{3 \rho}\left(x_{0}\right) \subset \Omega .
$$

Then, for every $f \in \mathrm{BMO}_{\varphi}(\Omega)$,

$$
\|f\|_{*, \varphi, \Omega^{\prime}} \leq c \eta(f, \varphi, \Omega)(2 \rho) .
$$

In particular, if $f \in \operatorname{VMO}_{\varphi}(\Omega)$, for every $\varepsilon>0$ there exist $\rho_{0}$ such that for every $\rho \leq \rho_{0}$ and $\Omega^{\prime}$ as above, we have

$$
\|f\|_{*, \varphi, \Omega^{\prime}}<\varepsilon .
$$

ii. If $\Omega^{\prime}$ is any $d$-regular subset of $\Omega$, then

$$
\|f\|_{*, \varphi, \Omega^{\prime}}<c\|f\|_{*, \varphi, \Omega} .
$$

iii. If sprtf $\subset B_{r}\left(x_{0}\right)$ with $B_{2 r}\left(x_{0}\right) \subset \Omega$, then

$$
\|f\|_{*, \varphi, \Omega} \leq c\|f\|_{*, \varphi, B_{2 r}} .
$$

iv. Let $B_{r}^{1}, B_{r}^{2}$ be a pair of (nondisjoint) balls of same radius, with $B_{4 r}^{1} \cup$ $B_{4 r}^{2} \subset \Omega$. Then for any $f \in B M O_{\varphi}(\Omega)$,

$$
\|f\|_{B M O_{\varphi}\left(B_{r}^{1} \cup B_{r}^{2}\right)} \leq c\left\{\|f\|_{B M O_{\varphi}\left(B_{4 r}^{1}\right)}+\|f\|_{B M O_{\varphi}\left(B_{4 r}^{2}\right)}\right\} .
$$


In the Euclidean case, for the standard $B M O$ space, (4.5) has been proved by [36] (see Lemma 2.1 p. 357).

Proof. Observe that (4.4) follows from (4.3). To prove (4.3), let $x \in \Omega^{\prime}$. Then $B_{2 \rho}(x) \supset \Omega^{\prime}$, hence

$$
\|f\|_{*, \varphi, \Omega^{\prime}}=\sup _{x \in \Omega^{\prime}, r \leq 2 \rho} \frac{1}{\left|B_{r}(x) \cap \Omega^{\prime}\right| \varphi(r)} \int_{B_{r} \cap \Omega^{\prime}}\left|f(y)-f_{B_{r} \cap \Omega^{\prime}}\right| d y .
$$

Now,

$$
\begin{aligned}
\frac{1}{\left|B_{r}(x) \cap \Omega^{\prime}\right| \varphi(r)} & \int_{B_{r} \cap \Omega^{\prime}}\left|f(y)-f_{B_{r} \cap \Omega^{\prime}}\right| d y \\
& \leq \frac{2}{\left|B_{r}(x) \cap \Omega^{\prime}\right| \varphi(r)} \int_{B_{r} \cap \Omega^{\prime}}\left|f(y)-f_{B_{r}}\right| d y \\
& \leq \frac{c}{\left|B_{r}(x)\right| \varphi(r)} \int_{B_{r}}\left|f(y)-f_{B_{r}}\right| d y \\
& \quad\left(\text { since } B_{r}(x) \subset B_{3 \rho}\left(x_{0}\right) \subset \Omega \text { for } r \leq 2 \rho,\right) \\
& \leq c \eta(f, \varphi, \Omega)(r) \leq c \eta(f, \varphi, \Omega)(2 \rho),
\end{aligned}
$$

that is (4.3).

To prove (4.5), pick $x \in \Omega^{\prime}$ and $r<\operatorname{diam}\left(\Omega^{\prime}\right)$. Since

$$
\left|B_{r}(x) \cap \Omega^{\prime}\right| \geq c\left|B_{r}(x)\right| \geq c\left|B_{r}(x) \cap \Omega\right|,
$$

we have

$$
\begin{aligned}
\frac{1}{\left|B_{r}(x) \cap \Omega^{\prime}\right| \varphi(r)} & \int_{B_{r} \cap \Omega^{\prime}}\left|f(y)-f_{B_{r} \cap \Omega^{\prime}}\right| d y \\
& \leq \frac{2}{\left|B_{r}(x) \cap \Omega^{\prime}\right| \varphi(r)} \int_{B_{r} \cap \Omega^{\prime}}\left|f(y)-f_{B_{r} \cap \Omega}\right| d y \\
& \leq \frac{c}{\left|B_{r}(x) \cap \Omega\right| \varphi(r)} \int_{B_{r} \cap \Omega}\left|f(y)-f_{B_{r} \cap \Omega}\right| d y \leq c\|f\|_{*, \varphi, \Omega} .
\end{aligned}
$$

To prove (4.6), pick $B_{s}(x)$ with $x \in \Omega$. If $x \notin B_{2 r}\left(x_{0}\right)$ the quantity

$$
\frac{1}{\left|B_{s}(x) \cap \Omega\right| \varphi(s)} \int_{B_{s}(x) \cap \Omega}\left|f(y)-f_{B_{s}(x) \cap \Omega}\right| d y
$$

does not vanish only if $B_{s}(x) \cap B_{r}\left(x_{0}\right) \neq \emptyset$, which implies $s>r$, but then 
it is bounded by

$$
\begin{aligned}
& \frac{2}{\left|B_{s}(x) \cap \Omega\right| \varphi(r)} \int_{B_{s}(x) \cap \Omega}\left|f(y)-f_{B_{r}}\right| d y \\
& \leq \frac{2}{\left|B_{s}(x) \cap \Omega\right| \varphi(r)}\left\{\int_{B_{r}}\left|f(y)-f_{B_{r}}\right| d y+\left|B_{s}(x) \cap \Omega \cap B_{r}^{c}\right| f_{B_{r}}|f(y)| d y\right\} \\
& \leq \frac{\left|B_{r}\right|}{\left|B_{s}(x) \cap \Omega\right|} \frac{2}{\varphi(r)} f_{B_{r}}\left|f(y)-f_{B_{r}}\right| d y+\frac{2}{\varphi(r)} f_{B_{r}}|f(y)| d y \\
& \leq c\left\{\|f\|_{*, \varphi, B_{r}}+\|f\|_{L^{1}\left(B_{r}\right)}\right\} \leq c\|f\|_{B M O_{\varphi}\left(B_{r}\right)}
\end{aligned}
$$

since

$$
\left|B_{s}(x) \cap \Omega\right| \geq\left|B_{r}(x) \cap \Omega\right| \geq c\left|B_{r}(x)\right| \geq c_{0}
$$

for $r$ fixed and $x$ ranging on $\Omega$.

On the other hand, if $x \in B_{2 r}$

$$
\begin{aligned}
& \frac{1}{\left|B_{s}(x) \cap \Omega\right| \varphi(s)} \int_{B_{s}(x) \cap \Omega}\left|f(y)-f_{B_{s}(x) \cap \Omega}\right| d y \\
& \leq \\
& \quad \frac{2}{\left|B_{s}(x) \cap B_{2 r}\left(x_{0}\right)\right| \varphi(s)} \int_{B_{s}(x) \cap B_{2 r}}\left|f(y)-f_{B_{s}(x) \cap B_{2 r}}\right| d y+ \\
& \quad+\frac{2}{\left|B_{s}(x) \cap \Omega\right| \varphi(s)} \frac{\left|B_{s}(x) \cap \Omega \cap B_{2 r}^{c}\right|}{\left|B_{s}(x) \cap B_{2 r}\right|} \int_{B_{r} \cap B_{s}(x)}|f(y)| d y \\
& \leq c\left\{\|f\|_{*, \varphi, B_{2 r}}+\|f\|_{1}\right\} \leq c\|f\|_{B M O_{\varphi}\left(B_{2 r}\right)} .
\end{aligned}
$$

The last inequality holds by the following argument:

if $B_{s}(x) \cap B_{r}=\emptyset$, then

$$
\int_{B_{r} \cap B_{s}(x)}|f(y)| d y=0 ;
$$

if $B_{s}(x) \cap B_{2 r}^{c}=\emptyset$, then

$$
\left|B_{s}(x) \cap \Omega \cap B_{2 r}^{c}\right|=0 ;
$$

if $B_{s}(x)$ intersects both $B_{r}$ and $B_{2 r}^{c}$, then $s>r / 2$ and $\left|B_{s}(x) \cap B_{2 r}\left(x_{0}\right)\right|$ is bounded away from zero; therefore

$$
\begin{aligned}
& \frac{2}{\left|B_{s}(x) \cap \Omega\right| \varphi(s)} \frac{\left|B_{s}(x) \cap \Omega \cap B_{2 r}^{c}\right|}{\left|B_{s}(x) \cap B_{2 r}\right|} \int_{B_{r} \cap B_{s}(x)}|f(y)| d y \\
& \leq \frac{2}{c \varphi(r / 2)} \int_{B_{r} \cap B_{s}(x)}|f(y)| d y \leq c\|f\|_{1} .
\end{aligned}
$$


To prove iv, let $\zeta_{i}(i=1,2)$ be smooth cutoff functions such that $\operatorname{sprt} \zeta_{i} \subset B_{2 r}^{i}, \zeta_{1}+\zeta_{2}=1$ in $B_{r}^{1} \cup B_{r}^{2}$. Then

$$
\begin{aligned}
\|f\|_{B M O_{\varphi}\left(B_{r}^{1} \cup B_{r}^{2}\right)} & \leq\left\|f \zeta_{1}\right\|_{B M O_{\varphi}\left(B_{r}^{1} \cup B_{r}^{2}\right)}+\left\|f \zeta_{2}\right\|_{B M O_{\varphi}\left(B_{r}^{1} \cup B_{r}^{2}\right)} \leq \\
& (\text { by }(4.5)) \\
& \leq c\left\{\left\|f \zeta_{1}\right\|_{B M O_{\varphi}\left(B_{4 r}^{1} \cup B_{4 r}^{2}\right)}+\left\|f \zeta_{2}\right\|_{B M O_{\varphi}\left(B_{4 r}^{1} \cup B_{4 r}^{2}\right)}\right\} \leq
\end{aligned}
$$$$
\text { (by }(4.6) \text { ) }
$$$$
\leq c\left\{\left\|f \zeta_{1}\right\|_{B M O_{\varphi}\left(B_{4 r}^{1}\right)}+\left\|f \zeta_{2}\right\|_{B M O_{\varphi}\left(B_{4 r}^{2}\right)}\right\} \leq
$$

(by Lemma 2.7)

$$
\leq c\left\{\|f\|_{B M O_{\varphi}\left(B_{4 r}^{1}\right)}+\|f\|_{B M O_{\varphi}\left(B_{4 r}^{2}\right)}\right\} \text {. }
$$

\subsection{Local estimates for uniformly hypoelliptic operators}

The main goal of this section is to prove local $\mathrm{BMO}_{\varphi}$-estimates for a class of "nonvariational uniformly hypoelliptic operators" that have been introduced by the authors in [4] and [5].

Let $X_{1}, X_{2}, \ldots, X_{q}$ be a system of smooth real vector fields defined and satisfying Hörmander's condition in a bounded domain $\Omega_{o}$ of $\mathbb{R}^{n}(n>q)$. Let $\Omega \subset \Omega_{o}$ be a $d$-regular domain (see $\S 4.1$ ). We introduce the Sobolev spaces $S^{2, p}$ induced by the vector fields $X_{i}$ by:

$$
\|f\|_{S^{2, p}}=\|f\|_{L^{p}}+\sum_{i=1}^{q}\left\|X_{i} f\right\|_{L^{p}}+\sum_{i, j=1}^{q}\left\|X_{i} X_{j} f\right\|_{L^{p}} .
$$

Let now $\varphi$ be as in $\S 1.2$ We say that $f \in S^{2, \varphi}(\Omega)$ if

$$
\|f\|_{S^{2, \varphi}(\Omega)}=\|f\|_{S^{2,1}(\Omega)}+\|f\|_{*, \varphi}+\sum_{i=1}^{q}\left\|X_{i} f\right\|_{*, \varphi}+\sum_{i, j=1}^{q}\left\|X_{i} X_{j} f\right\|_{*, \varphi}<\infty .
$$

Finally, for any norm $\|\cdot\|$ we will write:

$$
\begin{aligned}
\|D f\| & =\sum_{i=1}^{q}\left\|X_{i} f\right\| \\
\left\|D^{2} f\right\| & =\sum_{i, j=1}^{q}\left\|X_{i} X_{j} f\right\| .
\end{aligned}
$$

We can now state our main result: 
Theorem 4.5 Let $X_{1}, X_{2}, \ldots, X_{q}, \Omega$ be as above, and consider the differential operator

$$
\mathcal{L}=\sum_{i, j=1}^{q} a_{i j}(x) X_{i} X_{j}
$$

where the symmetric matrix $a_{i j}(x)$ satisfies the uniform ellipticity condition $\left(\right.$ on $\left.\mathbb{R}^{q}\right)$ :

$$
\mu|\xi|^{2} \leq \sum_{i, j=1}^{q} a_{i j}(x) \xi_{i} \xi_{j} \leq \mu^{-1}|\xi|^{2}
$$

for every $\xi \in \mathbb{R}^{q}$, a.e. $x \in \Omega$ and some positive constant $\mu$. Also, assume the coefficients $a_{i j}$ to be continuous and to belong to $V M O_{\widetilde{\varphi}}(\Omega)$ (where $\varphi$ and $\widetilde{\varphi}$ are defined as in §1.2). Then the following estimate holds:

$$
\|f\|_{S^{2, \varphi}\left(\Omega^{\prime}\right)} \leq c\left\{\|\mathcal{L} f\|_{S^{2, \varphi}(\Omega)}+\|f\|_{B M O_{\varphi}(\Omega)}\right\}
$$

for every $f \in S^{2, \varphi}(\Omega), \Omega^{\prime} \subset \subset \Omega, \Omega$ and $\Omega^{\prime}$ d-regular.

Remark 4.6 The assumption $a_{i j} \in V M O_{\widetilde{\varphi}}(\Omega)$ is fulfilled whatever $\varphi$ is if, for instance, $a_{i j}$ have a continuity modulus which, with respect to $d$, is $o\left(1 /|\log r|^{\alpha}\right)$ for every $\alpha>0$. For $\varphi=1$, it is enough to require the continuity modulus to be o $(1 /|\log r|)$. This, in turn, holds for instance if the $a_{i j}$ 's are Dini-continuous.

The proof of this result will be achieved throughout this section. We shall use many of the results proved by Rothschild and Stein [29] as well as some of the results in [4], [5]. From now on we shall therefore assume the reader familiar with [29]. Also, we will strictly follow notations and definitions as introduced in [5].

As a first step we apply Rothschild-Stein's "lifting theorem" (see Theorem 1.1. in [5]) to the vector fields $X_{i}$. We obtain new vector fields $\widetilde{X}_{i}$ which are free up to order $s$ and satisfy Hörmander's condition of step $s$, in some domain $\widetilde{\Omega} \subset \mathbb{R}^{N}$, with $N>n$. For $\xi=(x, t) \in \widetilde{\Omega}, x \in \mathbb{R}^{n}, t \in \mathbb{R}^{N-n}$, set $\widetilde{a}_{i j}(x, t)=a_{i j}(x)$ and let

$$
\widetilde{\mathcal{L}}=\sum_{i, j=1}^{q} \widetilde{a}_{i j}(\xi) \widetilde{X}_{i} \widetilde{X}_{j}
$$

be the lifted operator, defined in $\widetilde{\Omega}$.

Since most of the proof of Theorem 4.5 will involve the lifted vector fields $\widetilde{X}_{i}$ and the lifted operator $\widetilde{\mathcal{L}}$, living in $\mathbb{R}^{N}$, instead of the original vector fields $X_{i}$ and operator $\mathcal{L}$ living in $\mathbb{R}^{n}$, we change for a while our notation, denoting by $S^{2, p}, S^{2, \varphi}$, etc., the Sobolev spaces induced by the $\widetilde{X}_{i}$ 's, for functions defined in $\mathbb{R}^{N}$. 
The main steps of the proof of Theorem 4.5 are summarized in the following two theorems:

Theorem 4.7 There exists $r>0$ such that for any $f \in S^{2, \varphi}(\Omega), f$ compactly supported in some ball $B_{r} \subset \Omega$,

$$
\|f\|_{S^{2, \varphi}\left(B_{r}\right)} \leq c\left\{\|\widetilde{\mathcal{L}} f\|_{B M O_{\varphi}\left(B_{r}\right)}+\|f\|_{B M O_{\varphi}\left(B_{r}\right)}\right\} .
$$

Theorem 4.8 For every $f \in S^{2, \varphi}(\Omega), \Omega^{\prime} \subset \subset \Omega$,

$$
\|f\|_{S^{2, \varphi}\left(\Omega^{\prime}\right)} \leq c\left\{\|\widetilde{\mathcal{L}} f\|_{S^{2, \varphi}(\Omega)}+\|f\|_{B M O_{\varphi}(\Omega)}\right\} .
$$

The proof of Theorem 4.7 is based on a representation formula for second order derivatives of $f$, which is derived from the "parametrix formula" of Rothschild-Stein. To handle the variable coefficients $a_{i j}$ we use a version of "Korn's trick", which exploits the continuity of the coefficients (and represents one of the major differences from the line followed in [5]). The representation formula we get involves some integral operators, which we show to satisfy the assumptions of the theorems in $\S \S 2-3$.

Theorem 4.8 follows from Theorem 4.7 using cutoff functions and suitable interpolation inequalities for Sobolev norms. For technical reasons which will be explained later, the proof of these interpolation results significantly differs from the analogous estimate contained in [5].

\subsubsection{The parametrix formula}

Here we recall some facts and notation used in [5]; this paragraph can be skipped by the reader who is already familiar with this paper.

By Rothschild-Stein "approximation theorem" (see Theorem 1.6 in [5]), we can locally approximate the vector fields $\widetilde{X}_{i}$ with left invariant vector fields $Y_{i}$ defined on a suitable homogeneous group $G$ (which is actually $\mathbb{R}^{N}$ endowed with a suitable group structure). This approximation is expressed by the following identity which holds for every $f \in C_{0}^{\infty}(G)$ :

$$
\widetilde{X}_{i}\left(f\left(\Theta_{\xi}(\cdot)\right)\right)(\eta)=\left(Y_{i} f+R_{i}^{\xi} f\right)\left(\Theta_{\xi}(\eta)\right)
$$

where $\Theta_{\xi}(\eta)=\Theta(\xi, \eta)$ is a local diffeomorphism in $\mathbb{R}^{N}$, and the vector fields $R_{i}^{\xi}$ are remainders in a suitable sense (see [5]).

Next, we freeze $\widetilde{\mathcal{L}}$ at some point $\xi_{0} \in \widetilde{\Omega}$, and consider the frozen lifted operator:

$$
\widetilde{\mathcal{L}}_{0}=\sum_{i, j=1}^{q} \widetilde{a}_{i j}\left(\xi_{0}\right) \widetilde{X}_{i} \widetilde{X}_{j}
$$


To study $\widetilde{\mathcal{L}}_{0}$, we will consider the approximating operator, defined on $G$ :

$$
\mathcal{L}_{0}^{*}=\sum_{i, j=1}^{q} \widetilde{a}_{i j}\left(\xi_{0}\right) Y_{i} Y_{j}
$$

which, by [18], has a fundamental solution, denoted by $\Gamma\left(\xi_{0}, \cdot\right)$, which is homogeneous of degree $2-Q$, where $Q$ is the "homogeneous dimension" of $G$.

Let us recall the key definition which describes the singular and fractional integral operators which appear in this context.

Definition 4.9 We say that $k\left(\xi_{0} ; \xi, \eta\right)$ is a frozen kernel of type $\ell$, for some nonnegative integer $\ell$, if for every positive integer $m$ we can write

$k\left(\xi_{0} ; \xi, \eta\right)=\sum_{i=1}^{H_{m}} a_{i}(\xi) b_{i}(\eta)\left[D_{i} \Gamma\left(\xi_{0} ; \cdot\right)\right](\Theta(\eta, \xi))+a_{0}(\xi) b_{0}(\eta)\left[D_{0} \Gamma\left(\xi_{0} ; \cdot\right)\right](\Theta(\eta, \xi))$

where $a_{i}, b_{i}\left(i=0,1, \ldots H_{m}\right)$ are test functions, $D_{i}$ are differential operators such that: for $i=1, \ldots, H_{m}, D_{i}$ is homogeneous of degree $\leq 2-\ell$ (so that $D_{i} \Gamma\left(\xi_{0} ; \cdot\right)$ is a homogeneous function of degree $\left.\geq \ell-Q\right)$, and $D_{0}$ is a differential operator such that $D_{0} \Gamma\left(\xi_{0} ; \cdot\right)$ has $m$ derivatives with respect to the vector fields $Y_{i}(i=1, \ldots, q)$.

We say that $T\left(\xi_{0}\right)$ is a frozen operator of type $\ell \geq 1$ if $k\left(\xi_{0} ; \xi, \eta\right)$ is a frozen kernel of type $\ell$ and

$$
T\left(\xi_{0}\right) f(\xi)=\int k\left(\xi_{0} ; \xi, \eta\right) f(\eta) d \eta
$$

we say that $T\left(\xi_{0}\right)$ is a frozen operator of type 0 if $k\left(\xi_{0} ; \xi, \eta\right)$ is a frozen kernel of type 0 and

$$
T\left(\xi_{0}\right) f(\xi)=P . V . \int k\left(\xi_{0} ; \xi, \eta\right) f(\eta) d \eta+\alpha\left(\xi_{0}, \xi\right) f(\xi),
$$

where $\alpha$ is a bounded function, smooth in $\xi$ for any fixed $\xi_{0}$.

Next, we recall the basic "representation formula" which holds in this context (see Theorem 3.1 in [5]).

Theorem 4.10 (Parametrix for $\widetilde{\mathcal{L}}_{0}$ ) For every test function a, every $\xi_{0}$, there exist a frozen operator of type two, $P\left(\xi_{0}\right)$, and $q^{2}$ frozen operators of type one, $S_{i j}\left(\xi_{0}\right),(i, j=1, \ldots, q)$ such that for every test function $f$,

$$
P\left(\xi_{0}\right) \widetilde{\mathcal{L}}_{0} f(\xi)=a(\xi) f(\xi)+\sum_{i, j=1}^{q} \widetilde{a}_{i j}\left(\xi_{0}\right) S_{i j}\left(\xi_{0}\right) f(\xi) .
$$

We remark that the above formula holds for any compactly supported function $f \in S^{2, p}$, not necessarily smooth. 


\subsubsection{Representation formula for second order derivatives and proof of Theorem 4.7}

We are going to derive from (4.10) a representation formula for the second derivatives $\widetilde{X}_{i} \widetilde{X}_{j} f$ that will be the starting point to get our estimates. Note that in the following reasoning we will leave the point $\xi_{0}$ frozen.

Taking the derivative $\widetilde{X}_{i} \widetilde{X}_{j}$ of both sides of (4.10), writing $\widetilde{\mathcal{L}}_{0}=\widetilde{\mathcal{L}}+$ $\left(\widetilde{\mathcal{L}}_{0}-\widetilde{\mathcal{L}}\right)$ and exploiting the properties of the composition of differential operators with operators of type $\ell$ (see Lemma 2.9 and Theorem 2.10 in [5] for details), we get the following:

$$
\begin{aligned}
\widetilde{X}_{k} \widetilde{X}_{h}[a(\xi) f(\xi)]= & T\left(\xi_{0}\right) \widetilde{\mathcal{L}} f(\xi)+ \\
& +T\left(\xi_{0}\right)\left(\sum_{i, j=1}^{q}\left[\widetilde{a}_{i j}\left(\xi_{0}\right)-\widetilde{a}_{i j}(\cdot)\right] \widetilde{X}_{i} \widetilde{X}_{j} f\right)(\xi)+ \\
& -\sum_{i, j=1}^{q} \widetilde{a}_{i j}\left(\xi_{0}\right)\left(\sum_{l=1}^{q} T_{l}^{i j}\left(\xi_{0}\right) \widetilde{X}_{l} f(\xi)+T_{0}^{i j}\left(\xi_{0}\right) f(\xi)\right) .
\end{aligned}
$$

In the above formula, all the operators $T, T_{l}^{i j}$ are of type 0.

We are going to use the following fact, which will be proved at the end of this paragraph:

Lemma 4.11 If $T\left(\xi_{0}\right)$ is a frozen operator of type 0 or 1

$$
\left\|T\left(\xi_{0}\right) f\right\|_{B M O_{\varphi}\left(B_{r}\right)} \leq c\|f\|_{B M O_{\varphi}\left(B_{r}\right)}
$$

with $c$ independent of $\xi_{0}$.

In this context, the structure of space of homogeneous type which is more natural to consider, is that introduced by Rothschild and Stein, who defined the following quasidistance:

$$
d(\xi, \eta)=\left\|\Theta_{\xi}(\eta)\right\|
$$

where $\|\cdot\|$ is the homogeneous norm on $G$ (see [29]). Unlike the CarnotCarathéodory distance, introduced in $\S 4.1$, which is defined globally, $d$ is defined only locally. However, the two distances are locally equivalent, and it is therefore irrelevant to assume any of the two in the definition of the function spaces $B M O_{\varphi}, S^{2, \varphi}$, etc.; in particular, in view of this equivalence, we will also employ the results of $\S 4.1$. Also, we note that the RothschildStein's quasidistance $d$ is involved only in the proof of Lemma 4.11, which is established for small balls $B_{r}$. In the remaining parts of this section we shall use the Carnot-Carathéodory distance. 
Proof of Theorem 4.7 We start writing (4.11) for $f \in S_{\text {comp }}^{2, p}\left(B_{r}\left(\xi_{0}\right)\right)$, with $r$ to be chosen later, and $a \in C_{0}^{\infty}$ with $a \equiv 1$ on $B_{r}\left(\xi_{0}\right)$. Assume that the continuity moduli of $a_{i j}$ 's are bounded by $\omega(r)$. Then, taking $B M O_{\varphi}$ norms of both sides of (4.11), applying Lemma 4.11 and Theorem 2.4, we get:

$$
\begin{aligned}
\left\|\widetilde{X}_{k} \widetilde{X}_{h} f\right\|_{B M O_{\varphi}\left(B_{r}\right)} \leq c & \|\widetilde{\mathcal{L}} f\|_{B M O_{\varphi}\left(B_{r}\right)} \\
& +c \sum_{i, j=1}^{q}\left\{\left\|\widetilde{a}_{i j}\right\|_{*, \widetilde{\varphi}, B_{r}}+\omega(r)\right\}\left\|\widetilde{X}_{i} \widetilde{X}_{j} f\right\|_{B M O_{\varphi}\left(B_{r}\right)} \\
& +c\left\{\sum_{l=1}^{q}\left\|\widetilde{X}_{l} f\right\|_{B M O_{\varphi}\left(B_{r}\right)}+\|f\|_{B M O_{\varphi}\left(B_{r}\right)}\right\}
\end{aligned}
$$

where we used $\omega(r)$ to bound the term $\left\|\widetilde{a}_{i j}\left(\xi_{0}\right)-\widetilde{a}_{i j}(\cdot)\right\|_{L^{\infty}\left(B_{r}\right)}$ that comes from the multiplication Lemma.

Since $a_{i j} \in V M O_{\widetilde{\varphi}}(\Omega)$ with $\Omega \supset B_{3 r}$, in view of Lemma 4.4i, for $r$ small enough we can write, by (4.11):

$$
\begin{array}{r}
\left\|\widetilde{X}_{k} \widetilde{X}_{h} f\right\|_{B M O_{\varphi}\left(B_{r}\right)} \leq c\left\{\|\widetilde{\mathcal{L} f}\|_{B M O_{\varphi}\left(B_{r}\right)}+\sum_{l=1}^{q}\left\|\widetilde{X}_{l} f\right\|_{B M O_{\varphi}\left(B_{r}\right)}\right. \\
\left.+\|f\|_{B M O_{\varphi}\left(B_{r}\right)}\right\}
\end{array}
$$

Next, we want to get rid of the term $\left\|\widetilde{X}_{l} f\right\|_{B M O_{\varphi}\left(B_{r}\right)}$ in (4.13). To do this, we start again with (4.10), take only one derivative $\widetilde{X}_{i}$ and reason like above, getting:

$$
\begin{aligned}
\widetilde{X}_{k}[a(\xi) f(\xi)]=S\left(\xi_{0}\right) \widetilde{\mathcal{L}} f(\xi) & +S\left(\xi_{0}\right)\left(\sum_{i, j=1}^{q}\left[\widetilde{a}_{i j}\left(\xi_{0}\right)-\widetilde{a}_{i j}(\cdot)\right] \widetilde{X}_{i} \widetilde{X}_{j} f\right)(\xi) \\
& -\sum_{i, j=1}^{q} \widetilde{a}_{i j}\left(\xi_{0}\right) T^{i j}\left(\xi_{0}\right) f(\xi) .
\end{aligned}
$$

In the last formula, $S\left(\xi_{0}\right), T^{i j}\left(\xi_{0}\right)$ are, respectively, frozen operators of type 1,0 .

Taking $\mathrm{BMO}_{\varphi}$ norms in (4.13) and substituting in (4.12) we get

$$
\begin{aligned}
& \left\|\widetilde{X}_{k} \tilde{X}_{h} f\right\|_{B M O_{\varphi}\left(B_{r}\right)}+\left\|\widetilde{X}_{k} f\right\|_{B M O_{\varphi}\left(B_{r}\right)} \leq \\
& \quad \leq c\left\{\|\widetilde{\mathcal{L} f}\|_{B M O_{\varphi}\left(B_{r}\right)}+\|f\|_{B M O_{\varphi}\left(B_{r}\right)}\right\}+\varepsilon\left\|\widetilde{X}_{k} \widetilde{X}_{h} f\right\|_{B M O_{\varphi}\left(B_{r}\right)}
\end{aligned}
$$


with $\varepsilon$ small for small $r$. Hence we conclude:

$$
\|f\|_{S^{2, \varphi}\left(B_{r}\right)} \leq c\left\{\|\widetilde{\mathcal{L}} f\|_{B M O_{\varphi}\left(B_{r}\right)}+\|f\|_{B M O_{\varphi}\left(B_{r}\right)}\right\}
$$

for $r$ small enough.

Proof of Lemma 4.11 Let $T\left(\xi_{0}\right)$ be a frozen operator of type 0 . This means that:

$$
T\left(\xi_{0}\right) f(\xi)=\alpha\left(\xi_{0}\right) \beta(\xi) f(\xi)+P V \int k\left(\xi_{0} ; \xi, \eta\right) f(\eta) d \eta
$$

where $\alpha\left(\xi_{0}\right)$ is bounded and $\beta(\xi)$ is smooth, therefore, by Lemma 2.7, the operator

$$
f \mapsto \alpha\left(\xi_{0}\right) \beta f
$$

maps $\mathrm{BMO}_{\varphi}$ in itself continuously, uniformly in $\xi_{0}$; as to the singular kernel $k$, we can rewrite it in the following way:

$$
\begin{aligned}
& k\left(\xi_{0} ; \xi, \eta\right)=a(\xi) b(\eta)\left[D \Gamma\left(\xi_{0} ; \cdot\right)\right](\Theta(\eta, \xi))+ \\
& \quad+\sum_{i=1}^{H_{m}} a_{i}(\xi) b_{i}(\eta)\left[D_{i} \Gamma\left(\xi_{0} ; \cdot\right)\right](\Theta(\eta, \xi))+a_{0}(\xi) b_{0}(\eta)\left[D_{0} \Gamma\left(\xi_{0} ; \cdot\right)\right](\Theta(\eta, \xi))
\end{aligned}
$$

where:

- $a, b, a_{i}, b_{i}\left(i=0,1, \ldots H_{m}\right)$ are test functions;

- $D$ is a differential operator homogeneous of degree 2 , so that $D \Gamma\left(\xi_{0} ; \cdot\right)$ is a homogeneous function of degree $-Q$;

- $D_{i}$ are differential operators such that: for $i=1, \ldots, H_{m}, D_{i}$ is homogeneous of degree $<2$, so that $D_{i} \Gamma\left(\xi_{0} ; \cdot\right)$ is a homogeneous function of degree $>-Q)$;

- $D_{0}$ is a differential operator such that $D_{0} \Gamma\left(\xi_{0} ; \cdot\right)$ has $m$ derivatives with respect to the vector fields $Y_{i}(i=1, \ldots, q)$.

We now consider the most singular term:

$$
k(\xi, \eta)=a(\xi) b(\eta)\left[D \Gamma\left(\xi_{0} ; \cdot\right)\right](\Theta(\eta, \xi))
$$

and split it as follows:

$$
\begin{aligned}
k(\xi, \eta)= & \frac{a(\xi) b(\xi) c(\xi)\left[D \Gamma\left(\xi_{0} ; \cdot\right)\right](\Theta(\eta, \xi))}{g(\xi, \Theta(\xi, \eta))}+ \\
& +\frac{a(\xi) b(\xi)\left[D \Gamma\left(\xi_{0} ; \cdot\right)\right](\Theta(\eta, \xi))}{g(\xi, \Theta(\xi, \eta))}[g(\xi, \Theta(\xi, \eta))-c(\xi)]+ \\
& +a(\xi)[b(\eta)-b(\xi)]\left[D \Gamma\left(\xi_{0} ; \cdot\right)\right](\Theta(\eta, \xi)) \\
\equiv & k_{0}(\xi, \eta)+k_{1}(\xi, \eta)+k_{2}(\xi, \eta)
\end{aligned}
$$


where $g$ is the function appearing in the formula of change of variables (see Theorem 1.7 in [5]):

$$
u=\Theta(\xi, \eta) ; d \eta=g(\xi, u) d u ; g(\xi, u)=c(\xi)(1+O(\|u\|)) .
$$

The key properties of the kernels $k_{i}$ are: $k_{0}$ is singular, but satisfies a strong vanishing property, whereas $k_{1}, k_{2}$, are locally integrable, that is define fractional integral operators. Namely:

$$
\int_{r<\rho(\xi, \eta)<R} k_{0}(\xi, \eta) d \eta=a(\xi) b(\xi) c(\xi) \int_{r<\|u\|<R} Y_{i} Y_{j} \Gamma\left(\xi_{0} ; u\right) d u=0
$$

(see Theorem 2.4 in [5]) while

$$
\begin{aligned}
\frac{a(\xi) b(\xi)\left[D \Gamma\left(\xi_{0} ; \cdot\right)\right](\Theta(\eta, \xi))}{g(\xi, \Theta(\xi, \eta))} & {[g(\xi, \Theta(\xi, \eta))-c(\xi)]=} \\
& =\frac{a(\xi) b(\xi)\left[D \Gamma\left(\xi_{0} ; \cdot\right)\right](\Theta(\eta, \xi))}{1+O(\|\Theta(\xi, \eta)\|)} O(\|\Theta(\xi, \eta)\|)
\end{aligned}
$$

so that

$$
\left|k_{1}(\xi, \eta)\right| \leq \frac{c|a(\xi) b(\xi)|}{\|\Theta(\xi, \eta)\|^{Q-1}} .
$$

Finally, since $b$ is smooth and $\Theta$ is a diffeomorphism,

$$
\begin{aligned}
\mid a(\xi)[b(\eta)-b(\xi)] & {\left[D \Gamma\left(\xi_{0} ; \cdot\right)\right](\Theta(\eta, \xi)) \mid \leq } \\
& \leq c|a(\xi)||\eta-\xi|\left|D \Gamma\left(\xi_{0} ; \cdot\right)(\Theta(\eta, \xi))\right| \\
& \leq c|a(\xi)||(\Theta(\eta, \xi))|\left|D \Gamma\left(\xi_{0} ; \cdot\right)(\Theta(\eta, \xi))\right| \\
& \leq \frac{c|a(\xi)|}{\|\Theta(\xi, \eta)\|^{Q-1}} .
\end{aligned}
$$

Next, we claim that:

1. If $S\left(\xi_{0}\right)$ is a frozen operator of type $1, S\left(\xi_{0}\right)$ maps $B M O_{\varphi}\left(B_{r}\right)$ in itself continuously;

2. The operator $T_{0}$, defined by:

$$
T_{0} f(\xi)=P . V . \int k_{0}(\xi, \eta) f(\eta) d \eta,
$$

with $k_{0}(\xi, \eta)$ as above, maps $B M O_{\varphi}\left(B_{r}\right)$ in itself continuously.

Note that, by the above reasoning, the operator $T\left(\xi_{0}\right)$ can be written as $T_{0}$ plus a frozen operator of type 1 , plus a multiplication operator, which is continuous on $\mathrm{BMO}_{\varphi}$. Therefore the claim implies Lemma 4.11. 
Proof of the claim (sketch). By definition, a frozen operator of type 1 has a kernel which is the sum of several terms. Using Proposition 2.17 in [5], each of these terms can be proved to satisfy the assumptions of Theorem 3.5 (continuity of fractional integrals on $\mathrm{BMO}_{\varphi}$ ).

Analogously, the kernel $k_{0}$ in (4.15) can be proved to satisfy the assumption of Theorem 3.4 (continuity of singular integrals on $B M O_{\varphi}$ ). Note that the vanishing property of $k_{0}$ on spherical shells implies the vanishing property (3.10) required by Theorem 3.4.

Finally, we note that in both these estimates the constant is independent of $\xi_{0}$, according to the "uniform hypoellipticity estimate" contained in Theorem 2.2 of [4], which in turns relies on the ellipticity assumption on the matrix $a_{i j}$.

This completes the proof of Theorem 4.7.

\subsubsection{Interpolation inequalities and proof of Theorems 4.8 and 4.5}

The standard technique to get the interior estimate of Theorem 4.8 starting from the estimate for functions with small compact support (Theorem 4.7) requires cutoff functions and interpolation inequalities for Sobolev norms.

Lemma 4.12 For any $0<t<s, \xi \in \mathbb{R}^{N}$ there exists $\zeta \in C_{0}^{\infty}\left(\mathbb{R}^{N}\right)$ with the following properties:

i. $0 \leq \zeta \leq 1, \zeta \equiv 1$ on $B_{t}(\xi)$ and $\operatorname{sprt} \zeta \subseteq B_{s}(\xi)$;

ii.

$$
\left|D^{k} \zeta\right| \leq \frac{c}{(s-t)^{k}} \text { for } k=1,2,3
$$

where $D^{k}$ denotes any differential operator of the kind $\widetilde{X}_{i_{1}} \cdots \widetilde{X}_{i_{k}}$.

iii. For any $f \in B M O_{\varphi}$,

$$
\left\|f D^{k} \zeta\right\|_{*, \varphi} \leq \frac{c}{(s-t)^{k+1}}\|f\|_{B M O_{\varphi}} \text { for } k=0,1,2
$$

and $s-t$ small enough.

We will write

$$
B_{t}(\xi) \prec \zeta \prec B_{s}(\xi)
$$

to indicate that $\zeta$ satisfies all the previous properties. Note that i) and ii) can be accomplished as in [5], while property iii) follows by Lemma 2.7.

Comparing ii) and iii) we note that the regularity of $\zeta$ required by the multiplication theorem for $B M O_{\varphi}$, yields in iii the constant $\frac{c}{(s-t)^{k+1}}$, instead of $\frac{c}{(s-t)^{k}}$ which is typical in $L^{p}$ estimates. 
Theorem 4.13 (Interpolation inequality for Sobolev norms). For every $R>0, p \in(1, \infty), \varepsilon>0$, there exist positive constants $c, \alpha$ such that if $f \in S^{2, \varphi}\left(\mathbb{R}^{N}\right)$, $f$ compactly supported in $B_{R}(0)$, then

$$
\left\|\widetilde{X}_{i} f\right\|_{B M O_{\varphi}} \leq \varepsilon\|\Delta f\|_{B M O_{\varphi}}+\frac{c}{\varepsilon^{\alpha}}\|f\|_{B M O_{\varphi}}
$$

for every $i=1, \ldots, q$, where $\Delta f \equiv \sum_{i=1}^{q} \tilde{X}_{i}^{2} f$.

Proof. Let $\Delta^{*} \equiv \sum_{i=1}^{q} Y_{i}^{2}$ and let $\Gamma$ be the fundamental solution of $\Delta^{*}$, homogeneous of degree $(2-Q)$. Write $(4.10)$ with $\widetilde{\mathcal{L}}_{0}$ replaced by $\Delta$, a a test function equal to 1 on $B_{R}(0)$ and $f \in C_{0}^{\infty}\left(B_{R}(0)\right)$. We get:

$$
f(\xi)=P_{2} \Delta f(\xi)+S_{1} f(\xi)
$$

where $P_{2}, S_{1}$ are, respectively, constant operators of type 2, 1 (more precisely, they satisfy the definition of "frozen operators", with $\Gamma\left(\xi_{0} ; \cdot\right)$ replaced by $\left.\Gamma\right)$. Applying $\widetilde{X}_{i}$ to both sides of (4.10), we get

$$
\tilde{X}_{i} f(\xi)=P_{1} \Delta f(\xi)+S_{0} f(\xi)
$$

where $P_{1}, S_{0}$ are, respectively, a constant operator of type 1 and an operator of type 0 (in the same sense). Hence, in view of Theorem 3.6 of [5], the result will follow if we prove that, for any $\varepsilon>0$ small enough,

$$
\left\|P_{1} \Delta f\right\|_{*, \varphi} \leq \varepsilon\|\Delta f\|_{B M O_{\varphi}}+\frac{c}{\varepsilon^{\alpha}}\|f\|_{B M O_{\varphi}}
$$

Let $k(\xi, \eta)$ be the kernel of $P_{1}, \zeta_{\varepsilon}$ be a cutoff function with $B_{\varepsilon / 2}(\xi) \prec \zeta_{\varepsilon} \prec$ $B_{\varepsilon}(\xi)$, and let us split:

$$
\begin{aligned}
P_{1} \Delta f(\xi)= & \int_{\rho(\xi, \eta)>\varepsilon / 2} k(\xi, \eta)\left[1-\zeta_{\varepsilon}(\eta)\right] \Delta f(\eta) d \eta \\
& \quad+\int_{\rho(\xi, \eta) \leq \varepsilon} k(\xi, \eta) \Delta f(\eta) \zeta_{\varepsilon}(\eta) d \eta \\
= & I(\xi)+I I(\xi) .
\end{aligned}
$$

Then, integrating by parts

$$
\begin{aligned}
I(\xi) & =\int_{\rho(\xi, \eta)>\varepsilon / 2} \Delta^{T}\left(\left[1-\zeta_{\varepsilon}(\cdot)\right] k(\xi, \cdot)\right)(\eta) f(\eta) d \eta \\
& \equiv \int h^{\varepsilon}(\xi, \eta) f(\eta) d \eta
\end{aligned}
$$


with:

$$
\begin{gathered}
h^{\varepsilon}(\xi, \eta) \neq 0 \text { only if } \frac{\varepsilon}{2} \leq d(x, y) \leq \varepsilon ; \\
\left|h^{\varepsilon}\left(\xi_{1}, \eta\right)-h^{\varepsilon}\left(\xi_{2}, \eta\right)\right| \leq c(\varepsilon) d\left(\xi_{1}, \xi_{2}\right)^{\gamma} \text { for any } \xi_{1}, \xi_{2}
\end{gathered}
$$

From the above properties we have:

$$
\left|I\left(\xi_{1}\right)-I\left(\xi_{2}\right)\right| \leq c(\varepsilon) d\left(\xi_{1}, \xi_{2}\right)^{\gamma} \int|f(\eta)| d \eta .
$$

Hence $I(\xi)$ maps $L^{1}$ in $C^{\gamma}$ continuously, and therefore $B M O_{\varphi}$ in $B M O_{\varphi}$, with norm bounded by some $c(\varepsilon)$, which is readily seen to have the order of $\varepsilon^{-Q}$.

To bound $I I$, let

$$
T_{\varepsilon} g(\xi)=\int_{\rho(\xi, \eta)<\varepsilon} k(\xi, \eta) g(\eta) \zeta_{\varepsilon}(\eta) d \eta .
$$

The point is to prove that

$$
\left\|T_{\varepsilon} g\right\|_{B M O_{\varphi}} \leq c \varepsilon^{\gamma}\|g\|_{B M O_{\varphi}} \text { for some } \gamma>0 .
$$

(This, with $g=\Delta f$, will imply the desired result). Here we revise the proof of Theorem 3.5, with the kernel $k_{\alpha}(x, y)$ replaced by $k_{\varepsilon}(x, y)=$ $k(x, y) \zeta_{\varepsilon}(x, y), \zeta_{\varepsilon}(x, y)$ a smooth cutoff vanishing for $d(x, y)>\varepsilon$. Let $I_{\varepsilon}$ the fractional integral operator with kernel $k_{\varepsilon}$. As in the proof of Theorem 3.5, we split:

$$
\frac{1}{\varphi(r)} f_{B_{r}\left(x_{0}\right)}\left|I_{\varepsilon} f(x)-\tau\right| d x \equiv A+B
$$

Then, if $c r<\varepsilon$

$$
\begin{aligned}
|A| \leq & \frac{1}{\varphi(r)} f_{B_{r}\left(x_{0}\right)} d x \int_{d(x, y)<c r} \frac{|f(y)|}{|B(x ; y)|^{1-\alpha}} d y \leq \\
& \quad \text { as in the proof of Theorem 3.5) } \\
\leq & \omega(r)\|f\|_{*, \varphi} \\
& \quad(\text { for a suitable function } \omega(r) \rightarrow 0 \text { for } r \rightarrow 0) \\
\leq & \omega_{1}(\varepsilon)\|f\|_{*, \varphi}
\end{aligned}
$$

for another function $\omega_{1}(\varepsilon) \rightarrow 0$ for $\varepsilon \rightarrow 0$. If $c r \geq \varepsilon$

$$
|A| \leq \frac{c}{\varphi(\varepsilon)} f_{B_{r}\left(x_{0}\right)} d x \int_{d(x, y)<\varepsilon} \frac{|f(y)|}{|B(x ; y)|^{1-\alpha}} d y \leq \omega_{2}(\varepsilon)\|f\|_{*, \varphi}
$$

similarly to the proof of Theorem 3.5 , for some function $\omega_{2}(\varepsilon) \rightarrow 0$ for $\varepsilon \rightarrow 0$. 
To bound $B$ : if $c r>\varepsilon$ for a suitable $c$, then $B \equiv 0$. If $c r \leq \varepsilon$,

$$
\begin{aligned}
|B| & \leq \frac{c}{\varphi(r)} f_{B_{r}\left(x_{0}\right)} d\left(x_{0}, x\right)^{\beta} d x \int_{2 r \leq d\left(x_{0}, y\right)<c \varepsilon} \frac{\left|f(y)-f_{B_{2 r}}\right|+\left|f_{B_{2 r}}\right|}{\left|B\left(x_{0} ; y\right)\right|^{1-\alpha} d\left(x_{0}, y\right)^{\beta}} d y \\
& =D_{1}+D_{2} . \\
& D_{1} \leq c f_{B_{r}\left(x_{0}\right)} d\left(x_{0}, x\right)^{\beta} d x\left|B_{c \varepsilon}\left(x_{0}\right)\right|^{\alpha}\|f\|_{*, \varphi} r^{-\beta} \leq c \varepsilon^{\gamma}\|f\|_{*, \varphi} ;
\end{aligned}
$$

while, as in the proof of Theorem 3.5,

$$
D_{2} \leq \omega_{3}(r)\|f\|_{B M O_{\varphi}} \leq \omega_{4}(\varepsilon)\|f\|_{B M O_{\varphi}}
$$

for some functions $\omega_{3}(\varepsilon), \omega_{4}(\varepsilon)$, vanishing with $\varepsilon$. A careful reading of the proof of Theorem 3.5 shows that all the functions $\omega_{i}(\varepsilon)$ vanish at least as $\varepsilon^{\gamma}$ for some positive $\gamma$. This concludes the proof.

To handle functions which do not vanish at the boundary of the domain, we need a more flexible version of Theorem 4.13. However, the unpleasant presence, in the right-hand side of (4.16), of the exponent $\alpha$ (generally greater than 1), as well as the extra-singularity of the constant in Lemma 4.12 iii, with the subsequent lack of homogeneity in the interpolation inequality, forces us to modify the technique used in [4], [5].

By the way, we want to correct a minor mistake which occurs in [5], where we have stated an interpolation inequality for $S^{2, p}$ norms similar to Theorem 4.13, in the weaker form

$$
\|D f\|_{p} \leq \varepsilon\left\|D^{2} f\right\|_{p}+c(\varepsilon)\|f\|_{p} .
$$

Unfortunately, the subsequent arguments in [5] require $c(\varepsilon)$ to have the form $c / \varepsilon$; this sharper result can be actually proved with a slight modification of the proof.

First of all, we need the following technical lemma, which is adapted from [12], Lemma 4.1 p. 27.

Lemma 4.14 Let $\psi(t)$ be a bounded nonnegative function defined on the interval $\left[T_{0}, T_{1}\right]$, where $T_{1}>T_{0} \geq 0$. Suppose that for any $T_{0} \leq t<s \leq T_{1}, \psi$ satisfies

$$
\psi(t) \leq \vartheta \psi(s)+\frac{A}{(s-t)^{\alpha}}+B
$$

where $\vartheta, A, B, \alpha$ are nonnegative constants, and $\vartheta<\frac{1}{3}$. Then

$$
\psi(\rho) \leq c_{\alpha}\left[\frac{A}{(R-\rho)^{\alpha}}+B\right], \quad \forall T_{0} \leq \rho<R \leq T_{1}
$$

where $c_{\alpha}$ only depends on $\alpha$. 
Proof. Let $t_{0}=\rho, t_{i+1}=t_{i}+(1-\tau) \tau^{i}(R-\rho)(i=0,1,2, \ldots)$, where $0<\tau<1$ is to be determined. From (4.18)

$$
\psi\left(t_{i}\right) \leq \vartheta \psi\left(t_{i+1}\right)+\frac{A}{\left[(1-\tau) \tau^{i}(R-\rho)\right]^{\alpha}}+B \quad(i=0,1,2, \ldots) .
$$

By iteration,

$$
\psi\left(t_{0}\right) \leq \vartheta^{k} \psi\left(t_{k}\right)+\frac{A}{[(1-\tau)(R-\rho)]^{\alpha}} \sum_{i=0}^{k-1} \vartheta^{i} \tau^{-i \alpha}+B \sum_{i=0}^{k-1} \vartheta^{i} .
$$

Since $\vartheta<\frac{1}{3}$, we can choose $\tau$ such that $\vartheta \tau^{-\alpha}=\frac{1}{2}$; then

$$
\psi\left(t_{0}\right) \leq \vartheta^{k} \psi\left(t_{k}\right)+\frac{2}{\left[1-\left(\frac{2}{3}\right)^{1 / \alpha}\right]^{\alpha}} \cdot \frac{A}{(R-\rho)^{\alpha}}+\frac{3}{2} B .
$$

For $k \rightarrow+\infty$, we get (4.19).

We can now state our interpolation inequality for functions not necessarily vanishing at the boundary:

Theorem 4.15 For any $f \in S^{2, \varphi}\left(B_{R}\right), 0<\rho<R, \delta>0$

$$
\|D f\|_{B M O_{\varphi}\left(B_{\rho}\right)} \leq \delta\left\|D^{2} f\right\|_{B M O_{\varphi}\left(B_{R}\right)}+\frac{c_{\alpha}}{\delta^{\alpha}(R-\rho)^{2 \alpha}}\|f\|_{B M O_{\varphi}\left(B_{R}\right)}
$$

where $c_{\alpha}$ is independent of $f, \rho, R, \delta$, and $\alpha$ is as in Theorem 4.13.

Proof. If $f \in S^{2, \varphi}\left(B_{R}\right), 0<t<s \leq R$ and $\zeta$ is a cutoff function with $B_{t} \prec \zeta \prec B_{s}$, applying (4.16) to $f \zeta$, using Lemmas 4.4ii, 2.7 and 4.12, we get:

$$
\begin{aligned}
\|D f\|_{B M O_{\varphi}\left(B_{t}\right) \leq} \leq & \|D(\zeta f)\|_{B M O_{\varphi}\left(B_{s}\right)} \\
\leq & c_{1} \varepsilon\left\{\left\|\zeta D^{2} f\right\|_{B M O_{\varphi}\left(B_{s}\right)}+c\|D \zeta D f\|_{B M O_{\varphi}\left(B_{s}\right)}+\right. \\
& \left.\quad+c\left\|f D^{2} \zeta\right\|_{B M O_{\varphi}\left(B_{s}\right)}\right\}+\frac{c_{2}}{\varepsilon^{\alpha}}\|f\|_{B M O_{\varphi}\left(B_{s}\right)} \\
\leq & c_{1} \varepsilon\left\{\frac{1}{s-t}\left\|D^{2} f\right\|_{B M O_{\varphi}\left(B_{s}\right)}+\frac{1}{(s-t)^{2}}\|D f\|_{B M O_{\varphi}\left(B_{s}\right)}+\right. \\
& \left.\quad+\frac{1}{(s-t)^{3}}\|f\|_{B M O_{\varphi}\left(B_{s}\right)}\right\}+\frac{c_{2}}{\varepsilon^{\alpha}}\|f\|_{B M O_{\varphi}\left(B_{s}\right)} .
\end{aligned}
$$

Next, we pick $\varepsilon=\frac{\delta}{c_{1}}(s-t)^{2}$ :

$$
\begin{aligned}
\|D f\|_{B M O_{\varphi}\left(B_{t}\right)} \leq \delta & \left\{(s-t)\left\|D^{2} f\right\|_{B M O_{\varphi}\left(B_{s}\right)}+\|D f\|_{B M O_{\varphi}\left(B_{s}\right)}+\right. \\
& \left.+\frac{1}{(s-t)}\|f\|_{B M O_{\varphi}\left(B_{s}\right)}\right\}+\frac{c_{3}}{\delta^{\alpha}(s-t)^{2 \alpha}}\|f\|_{B M O_{\varphi}\left(B_{s}\right)} .
\end{aligned}
$$


Let $\psi(t)=\|D f\|_{B M O_{\varphi}\left(B_{t}\right)}$. Then

$$
\psi(t) \leq \delta \psi(s)+\frac{c_{4}}{\delta^{\alpha}(s-t)^{2 \alpha}}\|f\|_{B M O_{\varphi}\left(B_{R}\right)}+\delta\left\|D^{2} f\right\|_{B M O_{\varphi}\left(B_{R}\right)}
$$

for any $0<t<s<R$, and by Lemma 4.14 we get

$$
\psi(\rho) \leq c_{\alpha} \frac{c_{4}}{\delta^{\alpha}(R-\rho)^{2 \alpha}}\|f\|_{B M O_{\varphi}\left(B_{R}\right)}+c_{\alpha} \delta\left\|D^{2} f\right\|_{B M O_{\varphi}\left(B_{R}\right)}
$$

for any $0<\rho<R$.

Proof of Theorem 4.8. If $f \in S^{2, \varphi}(\Omega), B_{R} \subseteq \Omega,(R$ small enough to apply Theorem 4.7), $t<R, s=(t+R) / 2$, and $\zeta$ is a cutoff function, $B_{t} \prec \zeta \prec B_{s}$, we can apply (4.8) to $f \zeta$, getting

$$
\begin{aligned}
\left\|D^{2} f\right\|_{B M O_{\varphi}\left(B_{t}\right)} \leq & c\left\{\|\widetilde{\mathcal{L}}(f \zeta)\|_{B M O_{\varphi}\left(B_{s}\right)}+\|f \zeta\|_{B M O_{\varphi}\left(B_{s}\right)}\right\} \leq \\
& (\text { reasoning as above) } \\
\leq & c\left\{\frac{1}{s-t}\|\widetilde{\mathcal{L}} f\|_{B M O_{\varphi}\left(B_{s}\right)}+\frac{1}{(s-t)^{2}}\|D f\|_{B M O_{\varphi}\left(B_{s}\right)}+\right. \\
& \left.+\frac{1}{(s-t)^{3}}\|f\|_{B M O_{\varphi}\left(B_{s}\right)}\right\}+\frac{1}{s-t}\|f\|_{B M O_{\varphi}\left(B_{s}\right)} .
\end{aligned}
$$

Adding $\|D f\|_{B M O_{\varphi}\left(B_{t}\right)}$ to both sides, and applying Theorem 4.15 with $\delta=\varepsilon(s-t)^{2}$ and $\rho=s$, we get

$$
\begin{aligned}
\|f\|_{S^{2, \varphi}\left(B_{t}\right)} \leq c & \left\{\frac{1}{R-t}\|\widetilde{\mathcal{L}} f\|_{B M O_{\varphi}\left(B_{R}\right)}+\varepsilon\|f\|_{S^{2, \varphi}\left(B_{R}\right)}+\right. \\
& \left.+\frac{1}{\varepsilon^{\alpha}(R-t)^{4 \alpha+2}}\|f\|_{B M O_{\varphi}\left(B_{R}\right)}+\frac{1}{(R-t)^{3}}\|f\|_{B M O_{\varphi}\left(B_{R}\right)}\right\} .
\end{aligned}
$$

We fix $\varepsilon$ such that $c \varepsilon=\vartheta<\frac{1}{3}$, so that

$$
\begin{aligned}
\|f\|_{S^{2, \varphi}\left(B_{t}\right)} \leq \vartheta\|f\|_{S^{2, \varphi}\left(B_{R}\right)} & \\
& +\frac{c}{(R-t)^{4 \alpha+2}}\left\{R^{4 \alpha+1}\|\widetilde{\mathcal{L}} f\|_{B M O_{\varphi}\left(B_{R}\right)}+\|f\|_{B M O_{\varphi}\left(B_{R}\right)}\right\} .
\end{aligned}
$$

Finally, applying Lemma 4.14 we get

$$
\|f\|_{S^{2, \varphi}\left(B_{R / 2}\right)} \leq \frac{c}{R^{4 \alpha+2}}\left\{R^{4 \alpha+1}\|\widetilde{\mathcal{L}} f\|_{B M O_{\varphi}\left(B_{R}\right)}+\|f\|_{B M O_{\varphi}\left(B_{R}\right)}\right\} .
$$


for $R$ small enough. This implies (4.9), provided $\Omega^{\prime}$ is $d$-regular, by the following argument.

Let $\Omega^{\prime} \subset \bigcup_{i=1}^{n} B_{R / 4}^{i} \subset \bigcup_{i=1}^{n} B_{2 R}^{i} \subset \Omega$. Then:

$$
\begin{aligned}
\|f\|_{S^{2, \varphi}\left(\Omega^{\prime}\right)} & \leq c\|f\|_{S^{2, \varphi}\left(\cup B_{R / 4}^{i}\right)} \leq c \sum_{i=1}^{n}\|f\|_{S^{2, \varphi}\left(B_{R}^{i}\right)} \\
& \leq c \sum_{i=1}^{n}\left\{\|\widetilde{\mathcal{L}} f\|_{B M O_{\varphi}\left(B_{2 R}^{i}\right)}+\|f\|_{B M O_{\varphi}\left(B_{2 R}^{i}\right)}\right\} \\
& \leq c\left\{\|\widetilde{\mathcal{L} f}\|_{B M O_{\varphi}(\Omega)}+\|f\|_{B M O_{\varphi}(\Omega)}\right\}
\end{aligned}
$$

where: the first and last inequality are (4.5); the second is a consequence of (4.7), and the third is the estimate we have just proved. This completes the proof of Theorem 4.8 .

Conclusion of the proof of Theorem 4.5. Finally, from (4.9) we can easily come back to the original ("unlifted") variables, getting:

$$
\|f\|_{S^{2, \varphi}\left(\Omega^{\prime}\right)} \leq c\left\{\|\mathcal{L} f\|_{S^{2, \varphi}(\Omega)}+\|f\|_{B M O_{\varphi}(\Omega)}\right\}
$$

where now the function $f$ is defined on the domain $\Omega$ of $\mathbb{R}^{n}$, the function spaces $S^{2, \varphi}$ are defined with respect to the original vector fields $X_{i}$ and the metric induced by these vector fields. (See [5] p. 815 for the details.)

The possibility of comparing $B M O_{\varphi}$ norms in the "lifted" and "unlifted" context relies on Theorem 1.14 in [5]). This is exactly the assertion of Theorem 4.5.

\section{Appendix. The commutator theorem on $\mathrm{BMO}_{\varphi}$}

In this section we will describe a result which does not play directly any role in the previous parts of this paper but, as we have explained in the Introduction, partially motivates the assumptions we have made on the coefficients of the differential operator. Moreover, it can be of independent interest.

Let $X$ be a space of homogeneous type of finite measure and $K$ a Calderón-Zygmund operator on $X$ (see Definition 3.1) associated to a "standard kernel" $k$ satisfying (3.1), (3.2) and the following weak cancellation property:

$$
\left|\int_{d(x, y)>r} k(x, y) d y\right| \leq c
$$

independent of $x, r>0$. Moreover, assume that $k^{*}(x, y)=k(y, x)$ satisfies the same assumptions. 
Let $C_{a}$ be the commutator $\left[M_{a}, K\right]$,

$$
C_{a} f=a K f-K(a f) .
$$

In [6] it is proved that if $a \in B M O$, then $C_{a}$ is continuous on $L^{p}(X)$ for every $p \in(1, \infty)$, and

$$
\left\|C_{a} f\right\|_{p} \leq c\|a\|_{*}\|f\|_{p} .
$$

By Theorem 2.4, this also implies that, for $\varphi, \widetilde{\varphi}$ as in $\S 2.1$ and $a \in L^{\infty} \cap$ $B M O_{\widetilde{\varphi}}, C_{a}$ is bounded on $B M O_{\varphi}$ and

$$
\left\|C_{a} f\right\|_{B M O_{\varphi}} \leq c\left\{\|a\|_{\infty}+\|a\|_{*, \widetilde{\varphi}}\right\}\|f\|_{B M O_{\varphi}} .
$$

We are interested in proving a similar estimate, with a bound on $\left\|C_{a}\right\|$ independent of $\|a\|_{\infty}$; in other words, we want to prove that the operator norm of $C_{a}$ is small whenever the oscillation of $a$ (but not its absolute size) is small, in a suitable sense. This result extends, in the same spirit, the original commutator theorem due to Coifman-Rochberg-Weiss, see [15].

To get rid of the term $\|a\|_{\infty}$ in (5.2), we have to replace the seminorm $\|a\|_{*, \widetilde{\varphi}}$ with a stronger one, defined by a new function, even smaller than $\widetilde{\varphi}$ :

Theorem 5.1 Under the above assumptions, the following holds:

$$
\left\|C_{a} f\right\|_{B M O_{\varphi}} \leq c\|a\|_{*, \widehat{\varphi}}\|f\|_{B M O_{\varphi}}
$$

where

$$
\begin{gathered}
\widehat{\varphi}(r)=\frac{\varphi(r)}{1+S_{\varphi}(r)+\widehat{S}_{\varphi}(r)} \\
\widehat{S}_{\varphi}(r)=\int_{r}^{R} \frac{1}{t}\left(\int_{r}^{t} \frac{\varphi(s)}{s} d s\right) d t .
\end{gathered}
$$

Remark 5.2 For $\varphi=1$ we get the following result: if $a \in L^{2} M O$ (see $\left.\S 1.2\right)$, then $C_{a}$ maps $B M O$ in itself continuously, with $\left\|C_{a}\right\|$ bounded by the $L^{2} M O$ seminorm of a. As we have seen in §2.1, this assumption implies that a is a continuous function. More generally, it is easy to check that, under our assumptions, the function $\widehat{\varphi}$ always satisfies the bound

$$
\widehat{\varphi}(r) \leq \frac{c}{\log ^{2} r} \text { for small } r .
$$

This, in view of Spanne's criterion, implies that functions in $B M O_{\widehat{\varphi}}$ are always continuous (but not necessarily Hölder continuous, nor Dini continuous). Stegenga [33] and $\mathrm{Li}$ [24] have proved that if $a \in L^{\infty}$ and the multiplication operator $M_{a}$ is bounded on BMO, then $a \in L M O$. Since the continuity of the commutator implies the continuity of $M_{a}$ (and is a strictly stronger result), the assumption $a \in L^{2} M O$ is "almost necessary" for the commutator estimate. 
A complete proof of Theorem 5.1 is rather long and, essentially, does not introduce new ideas with respect to the techniques we have employed in sections 2-3. Therefore, for seek of brevity, we prefer to omit it. We only observe that to prove the theorem one has to estimate separately $\left\|C_{a} f\right\|_{*, \varphi}$ and $\left\|C_{a} f\right\|_{2}$. The assumptions on the "adjoint" kernel $k^{*}$ are involved only in the proof of the estimate

$$
\left\|C_{a} f\right\|_{2} \leq c\|a\|_{*}\|f\|_{2}
$$

given in [6].

\section{References}

[1] Agmon, S., Douglis, A. And Nirenberg, L.: Estimates near the boundary for solutions of elliptic partial differential equations satisfying general boundary conditions. I. Comm. Pure Appl. Math. 12 (1959), 623-727; II. Comm. Pure Appl. Math. 17 (1964), 35-92.

[2] Bellaïche, A.: The tangent space in sub-Riemannian geometry. In SubRiemannian geometry, 1-78. Progress in Mathematics 144. Birkhäuser, Basel, 1996.

[3] Bramanti, M.: Commutators of integral operators with positive kernels. Le Matematiche (Catania) 49 (1994), no. 1, 149-168.

[4] Bramanti, M. And Brandolini, L.: $L^{p}$-estimates for uniformly hypoelliptic operators with discontinuous coefficients on homogeneous groups. Rend. Sem. Mat. Univ. Politec. Torino 58 (2000), no. 4, 389-433.

[5] Bramanti, M. And Brandolini, L.: $L^{p}$-estimates for nonvariational hypoelliptic operators with VMO coefficients. Trans. Amer. Math. Soc. 352 (2000), no. 2, 781-822.

[6] Bramanti, M. and Cerutti, M. C.: Commutators of singular integrals on homogeneous spaces. Boll. Un. Mat. Ital. B (7) 10 (1996), no. 4, 843-883.

[7] Bramanti, M. and Cerutti, M. C.: Commutators of singular integrals and fractional integrals on homogeneous spaces. In Harmonic analysis and operator theory (Caracas, 1994), 81-94. Contemp. Math. 189. Amer. Math. Soc., Providence, RI, 1995.

[8] Burger, N.: Espace des fonctions à variation moyenne bornée sur un espace de nature homogène. C. R. Acad. Sci. Paris Sér. A-B 286 (1978), no. $3,139-142$.

[9] Chang, D.-C., Dafni, G. And Stein, E. M.: Hardy spaces BMO, and boundary value problems for the laplacian on a smooth domain in $R^{n}$. Trans. Amer. Math. Soc. 351 (1999), no. 4, 1605-1661.

[10] Chang, D.-C. And Li, S.-Y.: On the boundedness of multipliers, commutators and the second derivatives of Green's operators on $H^{1}$ and $B M O$. Ann. Scuola Norm. Sup. Pisa Cl. Sci. (4) 28 (1999), no. 2, 341-356. 
[11] Chang, D.-C., Krantz, S. and Stein, E. M.: $H^{p}$-theory on a smooth domain in $\mathbb{R}^{n}$ and elliptic boundary value problems. J. Funct. Anal. 114 (1993), no. 2, 286-347.

[12] Chen, Y.-Z. And Wu, L.-C.: Second Order Elliptic Equations and Elliptic Systems. Translations of Mathematical Monographs 174. American Mathematical Society, Providence, R.I., 1998.

[13] Chiarenza, F., Frasca, M. And Longo, P.: Interior $W^{2, p}$-estimates for nondivergence elliptic equations with discontinuous coefficients. Ricerche Mat. 40 (1991), no. 1, 149-168.

[14] Chiarenza, F., Frasca, M. and Longo, P.: $W^{2, p}$-solvability of the Dirichlet problem for non divergence elliptic equations with VMO coefficients. Trans. Amer. Math. Soc. 336 (1993), no. 2, 841-853.

[15] Coifman, R., Rochberg, R. and Weiss, G.: Factorization theorems for Hardy spaces in several variables. Ann. of Math. (2) 103 (1976), no. 3, 611-635.

[16] Coifman, R. And Weiss, G.: Analyse Harmonique Non-Commutative sur Certains Espaces Homogenes. Lecture Notes in Mathematics 242. SpringerVerlag, Berlin-Heidelberg-New York, 1971.

[17] Fefferman, C. And Phong, D. H.: Subelliptic eigenvalue problems. In Conference on harmonic Analysis in honor of Antoni Zygmund (Chicago, Ill., 1981), 590-606. Wadsworth Math. Ser. Wadsworth, Belmont, 1983.

[18] Folland, G. B.: Subelliptic estimates and function spaces on nilpotent Lie groups. Ark. Mat. 13 (1975), no. 2, 161-207.

[19] Garofalo, N. And Nhieu, D.-M.: Isoperimetric and Sobolev inequalities for Carnot-Carathéodory Spaces and the Existence of Minimal Surfaces. Comm. Pure Appl. Math. 49 (1996), no. 10, 1081-1144.

[20] Gilbarg, D. and Trudinger, N. S.: Elliptic Partial Differential Equations of Second Order. Second Edition. Springer-Verlag, Berlin-HeidelbergNew York-Tokio, 1983.

[21] Harboure, E., Segovia, C. And Torrea, J. L.: Boundedness of commutators of fractional and singular integrals for the extreme values of $p$. Illinois J. Math. 41 (1997), no. 4, 676-700.

[22] John, F. And Nirenberg, L.: On functions of bounded mean oscillation. Comm. Pure Appl. Math. 14 (1961), 175-188.

[23] Korn, A.: Zwei Anwendungen der Methode der sukzesivven Annäherungen. In Schwarz Festschrift, 215-229. Julius Springer, Berlin, 1914.

[24] LI, S.-Y.: Toeplitz Operators on Hardy space $H^{p}(S)$ with $0<p \leq 1$. Integral Equations Operator Theory 15 (1992), no. 5, 807-824.

[25] Macias, R. A. And Segovia, C.. Lipschitz Functions on Spaces of Homogeneous Type. Adv. in Math. 33 (1979), no. 3, 257-270. 
[26] Nagel, A., Stein, E. M. And Wainger, S.: Balls and metrics defined by vector fields I: Basic properties. Acta Math. 155 (1985), no. 1-2, 103-147.

[27] Peetre, J.: On convolution operators leaving $L^{p, \lambda}$ spaces invariant. Ann. Mat. Pura Appl. (4) 72 (1966), 295-304.

[28] PÉREz, C.: Endpoint estimates for commutators of singular integral operators. J. Funct. Anal. 128 (1995), no. 1, 163-185.

[29] Rothschild, L. P. And Stein, E. M.: Hypoelliptic differential operators and nilpotent groups. Acta Math. 137 (1976), no. 3-4, 247-320.

[30] SÁnchez-Calle, A.: Fundamental solutions and geometry of sum of squares of vector fields. Invent. Math. 78 (1984), no. 1, 143-160.

[31] Sarason, D.: Functions of vanishing mean oscillations. Trans. Amer. Math. Soc. 207 (1975), 391-405.

[32] Spanne, S.: Some function spaces defined using the mean oscillation over cubes. Ann. Scuola Norm. Sup. Pisa (3) 19 (1965), 593-608.

[33] Stegenga, D. A.: Bounded Toeplitz operators on $H^{1}$ and applications of the duality between $H^{1}$ and the functions of bounded mean oscillation. Amer. J. Math. 98 (1976), no. 3, 573-589.

[34] Stein, E. M.: Harmonic Analysis: Real-Variable Methods, Orthogonality and Oscillatory Integrals. Princeton Univ. Press. Princeton, New Jersey, 1993.

[35] Torchinsky, A.: Real-Variable Methods in Harmonic Analysis. Pure and Applied Mathematics 123. Academic Press, Orlando, 1986.

[36] Transirico, M., Troisi, M. and Vitolo, A.: BMO spaces on domains of $\mathbb{R}^{n}$. Ricerche Mat. 45 (1996), no. 2, 355-378.

Recibido: 21 de abril de 2003

Marco Bramanti

Dipartimento di Matematica

Politecnico di Milano

Via Bonardi 9

20133 Milano, Italy

marbra@mate.polimi.it

Luca Brandolini

Dipartimento di Ingegneria Gestionale e dell'Informazione

Università degli Studi di Bergamo

Viale G. Marconi 5

24044 Dalmine BG, Italy

luca.brandolini@unibg.it 